UNITU-THEP-9/1997

hep-ph/9705267

TU-GK-97-003

\title{
Nucleon Form Factors in a Covariant Diquark-Quark Model ${ }^{\dagger}$
}

\author{
G. Hellstern, R. Alkofer, M. Oettel and H. Reinhardt \\ Institute for Theoretical Physics, Tübingen University \\ Auf der Morgenstelle 14, D-72076 Tübingen, Germany
}

\begin{abstract}
In a model where constituent quarks and diquarks interact through quark exchange the Bethe-Salpeter equation in ladder approximation for the nucleon is solved. Quark and diquark confinement is effectively parametrized by choosing appropriately modified propagators. The coupling to external currents is implemented via nontrivial vertex functions for quarks and diquarks to ensure gauge invariance at the constituent level. Nucleon matrix elements are evaluated in a generalised impulse approximation, and electromagnetic, pionic and axial form factors are calculated.
\end{abstract}

Key words: Baryon structure; Diquarks; Bethe-Salpeter equation; Form Factors.

PACS: 14.20.DH, 12.39.Ki, 12.40.Yx, 13.40.Gp, 11.10.St

†Supported by COSY under contract 41315266, BMBF under contract 06TU888 and Graduiertenkolleg "Hadronen und Kerne" (DFG Mu705/3). 


\section{Introduction}

Accelerators like ELSA, CEBAF and COSY will investigate hadron observables at a scale intermediate to the low-energy region where hadron phenomena mainly reflect the underlying principles of chiral symmetry and its dynamical breakdown, and to the largemomentum regime where the "strong" interaction has the appearance of being a perturbation on free-moving quarks and gluons. A theoretical description of the corresponding intermediate energy physics aims at an understanding of the interplay between hadronic degrees of freedom and their intrinsic quark substructure. At present the description of the intermediate energy region of several $\mathrm{GeV}$, however, requires to use an effective parametrization of confinement within a fully relativistic formalism. Furthermore, in the description of hadrons as bound states of quarks nonperturbative methods are unavoidable. On the other hand, for large momentum transfers the perturbative results of QCD should be met. In this paper we set up a further step in the development of an interpolating model to describe baryon structure in the intermediate energy region.

The basic idea of this model is to parametrize complicated and/or unknown structures within baryons by means of two-quark correlations in the overall antisymmetrized colorantitriplet channels. This amounts to describing baryons effectively as bound states of constituent quarks and diquarks (for a recent digest of diquark models see e.g. [四]). Guided by the notion that a fully relativistic Faddeev equation determines baryons as bound states of three quarks and provides a complete and correct picture of baryons we will study the Bethe-Salpeter equation for a diquark-quark system. The minimal physics which has to be implemented in such a picture is to allow the diquark to couple to two quarks. Such a coupling gives rise to quark exchange between the diquark and the "third" quark within the baryon [2,3]. In this first investigation we will restrict ourselves to this minimal picture. Note that quark exchange is also required to reinstate the Pauli principle within a Faddeev approach using effective diquarks.

A further important ingredient of our model is the effective parametrization of confinement for the constituents of the baryon, the quarks and diquarks. This is achieved by modifying the propagators of these particles such that no Lehmann representation can be found for these propagators. The price which has to be paid is an essential singularity at timelike infinite momentum carried by these propagators $\square$. Note that such a singularity prohibits the use of dispersion relations. Nevertheless, we believe that the benefits of such a description, the absence of unphysical thresholds, is worth the loss of applicability of dispersion relations.

Given the fact that there exists numerous models for baryons, different types of bag [5] and soliton [6,7] models as well as non-relativistic 2 [8] [10] and relativistic potential

1 Recent studies of a confining interaction within the Klein-Gordon equation revealed an essential singularity in the Isgur-Wise function of heavy quarks [4].

2 For a full solution of the nonrelativistic Faddeev equation see ref. [11]. These authors, however, conclude: "As a result any nonrelativistic constituent quark model can at most be considered as a parametrization of the baryon energy levels, rather than as a dynamical model for light three quarks systems. Certainly it will not prove acceptable for future applications such as the 
models [12], one is tempted to argue that there is no need for another baryon model. A closer inspection, however, reveals that most, if not all, of these models display severe shortcomings when applied to intermediate energy reactions. Furthermore, at low energies these models describe e.g. the nucleon reasonably but not overwhelmingly well. These remark applies to models being so different as for example the Skyrmion versus the MIT bag. Hybrid models like the chiral bag [13,14] have deepened our insight but have not been able to answer the question of the low-energy structure of the nucleon conclusively. A very recent effort to understand quark correlations in a solitonic background on the basis of the Nambu-Jona-Lasinio model [15] have revealed that, at least within this model, quark binding energies due to a self-consistent solitonic background and due to direct two-quark and three-quark correlations are of the same order of magnitude [16. Therefore, one might argue that low-energy observables are not sufficient to determine the structure of baryons even qualitatively. On the other hand, high precision data in a momentum regime where quarks certainly are dominant over collective (solitonic) effects but interactions are still highly non-perturbative will help to clarify issues related to the structure of baryons. The interpretation of these data, on the other hand, requires a not too complicated modelling of the corresponding reactions. In view of these remarks, we believe that there is still need for a baryon model adapted to the intermediate energy regime.

This paper is organized as follows: In sect. 2 we describe our model: Constituent quarks and diquarks interact via a Yukawa interaction which represents the fact that a diquark may decay into two quarks. We discuss a modification of the quark and diquark propagators which allow to mimic confinement efficaciously. In sect. 3 we discuss the diquarkquark Bethe-Salpeter equation for the nucleon. Special emphasis is hereby put on the decomposition in the Dirac algebra. The components of the nucleon spinor are expanded in hyperspherical harmonics. Numerical solutions including scalar diquarks are presented. In sect. D various nucleon form factors are calculated using Mandelstam's formalism. The vertex functions of external currents to quarks and diquarks are constructed from the corresponding Ward identities such that gauge invariance and symmetries are respected at the constituent level. In sect. 5 we summarize the results obtained so far and give an outlook.

\section{The Model}

As noted in the introduction the basic idea of our model is to parametrize complicated and/or unknown structures within baryons by means of two-quark correlations in the overall antisymmetrized color-antitriplett channels. Furthermore, it is assumed that scalar $\left(0^{+}\right)$and axialvector $\left(1^{+}\right)$diquarks are sufficient to describe baryons. As we are interested mainly in the nucleon we will only consider two flavors. Due to the Pauli principle the scalar and axialvector diquarks are then isoscalar and isovector, respectively. The minimal physics which has to be implemented in such a picture is to allow the diquark to couple

description of electromagnetic form factors, hadronic decays, and other dynamical observables that are determined by the behaviour of the baryon wave function and are generally much influenced by quark-quark potential parameters." 
to two quarks. Such a coupling gives rise to quark exchange between the diquark and the "third" quark within the baryon. In this first investigation we will restrict ourselves to this minimal picture. Note also that quark exchange is required to reinstate the Pauli principle on the three-quark level within a Faddeev approach using effective diquarks [3].

Additionally, we want to modify the kinetic terms of the fields in a way that will allow for an effective parametrization of confinement. We will do this on the Lagrangian level only symbolically, and discuss the specific form of these terms in connection with the propagators used in the Bethe-Salpeter equation.

The above description may be formalized with the help of the following Lagrangian:

$$
\begin{aligned}
\mathcal{L}= & \bar{q}_{A}(x)\left(i \gamma^{\mu} \partial_{\mu}-m_{q}\right) f\left(-\partial^{2} / m_{q}^{2}\right) q_{A}(x)+\Delta_{A}^{\dagger}(x)\left(-\partial_{\mu} \partial^{\mu}-m_{s}^{2}\right) f\left(-\partial^{2} / m_{s}^{2}\right) \Delta_{A}(x) \\
& -\frac{1}{4} F_{\mu \nu}^{\dagger}(x) f\left(-\partial^{2} / m_{a}^{2}\right) F^{\mu \nu}(x)+\frac{1}{2} m_{a}^{2} \Delta_{A a \mu}^{\dagger}(x) f\left(-\partial^{2} / m_{a}^{2}\right) \Delta_{A a}^{\mu}(x) \\
& +\frac{\epsilon^{A B C}}{\sqrt{2}}\left(g_{s} q_{C}^{T}(x) C i \gamma^{5} \frac{t_{\mathcal{A}}}{2} q_{B}(x) \Delta_{A}^{*}(x)+g_{s}^{*} \Delta_{A}(x) \bar{q}_{B}(x) i \gamma^{5} C \frac{t_{\mathcal{A}}}{2} \bar{q}_{C}^{T}(x)\right) \\
& +\frac{\epsilon^{A B C}}{\sqrt{2}}\left(g_{a} q_{C}^{T}(x) C \frac{i \gamma^{\mu}}{\sqrt{2}} \frac{t_{\mathcal{S}}^{a}}{2} q_{B}(x) \Delta_{A a \mu}^{*}(x)-g_{a}^{*} \Delta_{A a \mu}(x) \bar{q}_{B}(x) \frac{t_{\mathcal{S}}^{a}}{2} \frac{i \gamma^{\mu}}{\sqrt{2}} C \bar{q}_{C}^{T}(x)\right) .
\end{aligned}
$$

Here $q(x)$ describes the constituent quark with mass $m_{q}$. The scalar and axialvector diquark field are called $\Delta$ and $\Delta^{\mu}$, respectively; their masses are denoted by $m_{s}$ and $m_{a}$. The diquark field strength tensor is given by $F^{\mu \nu}=\partial^{\mu} \Delta^{\nu}-\partial^{\nu} \Delta^{\mu}+\left[\Delta^{\mu}, \Delta^{\nu}\right]$ as the axialvector diquark field is due to its isospin of non-abelian nature. Note, however, that we will not take into account the related self-interactions of this field. The function $f$ stands as reminder that the tree-level propagators of these fields will be modified in order to describe their confinement. The Yukawa couplings between quarks and diquarks are denoted by $g_{s}$ and $g_{a}$. Note that these interactions are renormalizable. Nevertheless, we will eventually substitute $g_{s}$ and $g_{a}$ by some additional momentum-dependent factors in order to study the influence of the fact that realistic diquarks are certainly not pointlike.

Writing down the Yukawa interactions there are some ambiguities concerning the phases of the interaction term. As we have chosen a hermitian interaction term we expect the coupling constants to be real. The notation using $g_{s, a}$ and $g_{s, a}^{*}$ is nevertheless chosen in order to allow for a more complete discussion.

In the Lagrangian (1) color indices are denoted by capital and isospin indices by small letters. The color coupling via the $\epsilon$-tensor is determined by the assumed color antitriplet nature of the diquark allowing for a color singlet interaction term. The matrix $t_{\mathcal{A}}=\tau^{2}$ is the antisymmetric generator of the isospin group, the matrices $t_{\mathcal{S}}^{a}=\left\{\tau^{3}, \mathbf{1}, \tau^{1}\right\}=\left\{\boldsymbol{\tau} \tau^{2}\right\}$ are the symmetric generators.

As we will use the Bethe-Salpeter equation in Euclidean space we will perform a Wick

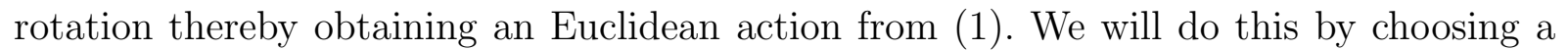
positive definite metric and hermitian Dirac matrices, $\left\{\gamma_{\mu}, \gamma_{\nu}\right\}=2 \delta_{\mu \nu}$ and $\gamma_{\mu}^{\dagger}=\gamma_{\mu}$. 
In order to mimic confinement we will choose the function $f$ in the Lagrangian (11) to be

$$
f(x)=1-e^{-d(1+x)},
$$

i.e. the (Euclidean) quark and diquark propagators are given by

$$
\begin{aligned}
S(p) & =\frac{i p-m_{q}}{p^{2}+m_{q}^{2}}\left(1-e^{-d\left(p^{2}+m_{q}^{2}\right) / m_{q}^{2}}\right) \\
D_{A B}(p) & =-\frac{\delta^{A B}}{p^{2}+m_{s}^{2}}\left(1-e^{-d\left(p^{2}+m_{s}^{2}\right) / m_{s}^{2}}\right), \\
D_{A a B b}^{\mu \nu}(p) & =-\frac{\delta^{A B} \delta^{a b}\left(\delta^{\mu \nu}+p^{\mu} p^{\nu} / m_{a}^{2}\right)}{p^{2}+m_{a}^{2}}\left(1-e^{-d\left(p^{2}+m_{a}^{2}\right) / m_{a}^{2}}\right) .
\end{aligned}
$$

Obviously, $f$ modifies these propagators as compared to the ones for free Dirac, KleinGordon and Proca particles. It is chosen such that it removes the free particle pole at $p^{2}=-m_{i}^{2}, i=q, s, a$ While $d=1$ is the minimal way to mimic confinement, we will also study the cases $d>1$ where the propagators are less modified for spacelike momenta. The propagators (3, (3, 5) are free of singularities for all finite $p^{2}$. However, they possess an essential singularity at timelike infinite momenta, $p^{2}=-\infty$. There exists no Lehmann representation for these propagators: They describe confined particles. With respect to our calculations the interesting property is simply the non-existence of thresholds related to diquark and quark production. 3

The form of the propagators (3, 4, 5) is motivated from studies in the Munzcek-Nemirovsky model [17]. In this model the effective low-energy quark-quark interaction is modelled as $\delta$-function in momentum space. These leads to a quark propagator without poles on the real $p^{2}$ axis and a behaviour for large negative $p^{2}$ similar to the one of (3) 4. Recently, it has been shown that within this model diquarks are also confined if one includes a non-trivial quark-gluon vertex [19]. The diquark propagator does not have a pole and is indeed similar to the form (4, 5) whereas meson masses as given by poles of the corresponding propagators stay almost unaltered as compared to the model with a tree level quark-gluon vertex. Thus, these investigations give us some confidence that an effective description of confinement via the parametrizations (3,4.5) is indeed sensible. Nevertheless, for comparison we will also use in the following free tree-level propagators, i.e. $f \equiv 1$.

As the color structure of the propagators is trivial, color indices will be suppressed in the following. In this paper we will assume isospin symmetry and therefore also suppress isospin indices.

$\overline{3}$ Note that these form of the propagators prohibits the use of dispersion relations. This is unavoidable because unitarity is violated by construction.

4 Note that an infrared cutoff in the NJL model has similar effects [18]. 


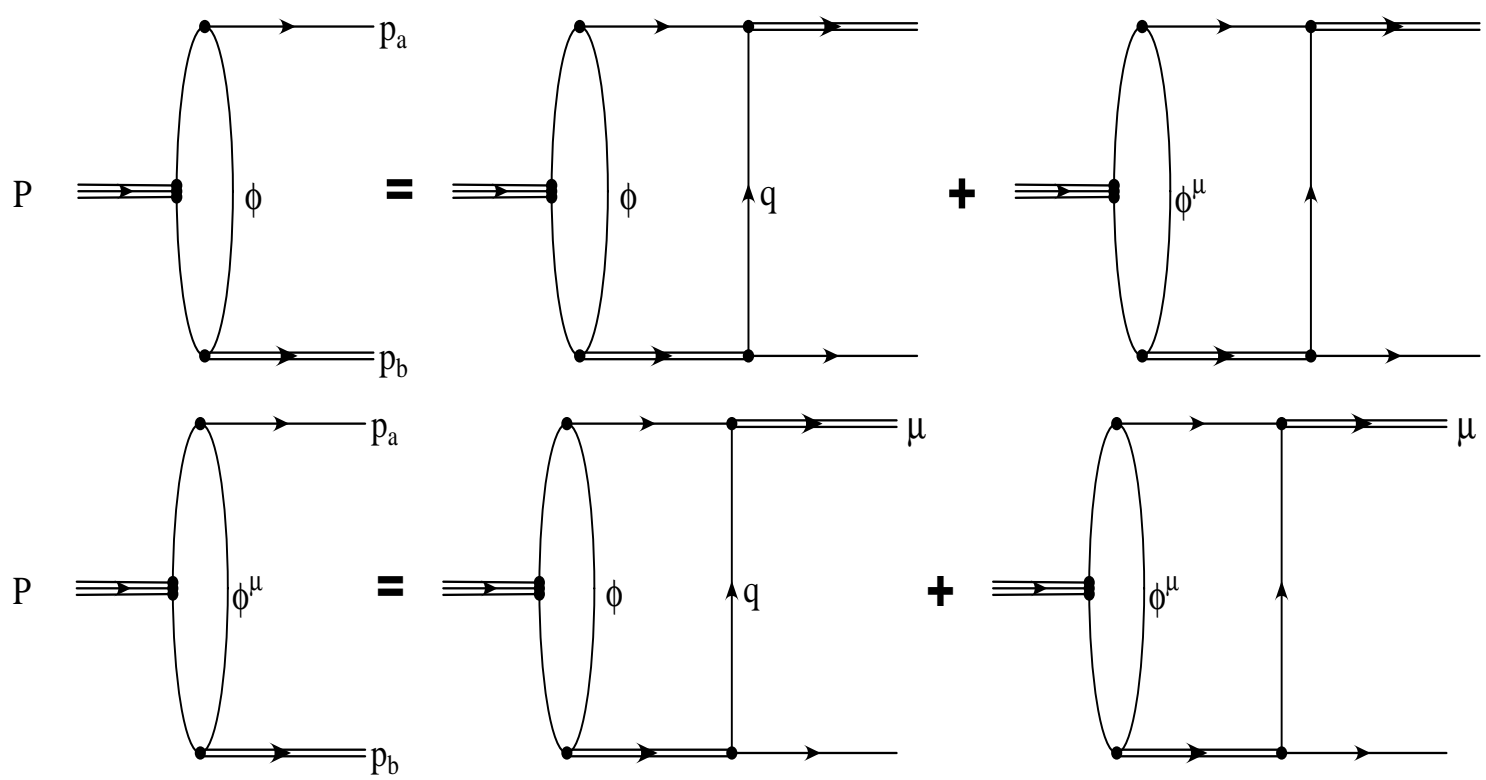

Fig. 1. The Bethe-Salpeter equation for the scalar and axial nucleon vertex functions.

\section{Diquark-Quark Bethe-Salpeter Equation for the Nucleon}

Using the Lagrangian (1) the Bethe-Salpeter equation for the diquark-quark bound state vertex functions is given by

$$
\begin{aligned}
\Phi(p)= & -\left|g_{s}\right|^{2} \int \frac{d^{4} p^{\prime}}{(2 \pi)^{4}} \gamma_{5} S(-q) \gamma_{5} S\left(p_{a}^{\prime}\right) D\left(p_{b}^{\prime}\right) \Phi\left(p^{\prime}\right) \\
& +\sqrt{\frac{3}{2}} g_{s}^{*} g_{a} \int \frac{d^{4} p^{\prime}}{(2 \pi)^{4}} \gamma^{\mu} S(-q) \gamma_{5} S\left(p_{a}^{\prime}\right) D^{\mu \nu}\left(p_{b}^{\prime}\right) \Phi^{\nu}\left(p^{\prime}\right), \\
\Phi^{\mu}(p)= & -\sqrt{\frac{3}{2}} g_{s} g_{a}^{*} \int \frac{d^{4} p^{\prime}}{(2 \pi)^{4}} \gamma_{5} S(-q) \gamma^{\mu} S\left(p_{a}^{\prime}\right) D\left(p_{b}^{\prime}\right) \Phi\left(p^{\prime}\right) \\
& -\left|g_{a}\right|^{2} \int \frac{d^{4} p^{\prime}}{(2 \pi)^{4}} \gamma^{\lambda} S(-q) \gamma^{\mu} S\left(p_{a}^{\prime}\right) D^{\lambda \rho}\left(p_{b}^{\prime}\right) \Phi^{\rho}\left(p^{\prime}\right) .
\end{aligned}
$$

These equations are diagrammatically represented in fig. 1. Hereby $P=p_{a}+p_{b}$ denotes the total momentum of the bound state and $p$ or $p^{\prime}$ is the relative momentum between quark and diquark:

$$
p=(1-\eta) p_{a}-\eta p_{b}
$$

The physical solution does not depend on the parameter $\eta \in[0,1]$, however, as it is known from previous studies of Bethe-Salpeter equations the accuracy of the numerical solution may depend on it (see e.g. [20]). In these first exploratory calculations we will only use the choice which renders the equations $(6,7)$ as simple as possible, $\eta=1 / 2$.

Using a static (momentum independent) approximation for the quark exchange, the Bethe-Salpeter equation (6, 7) can be reduced to a Dirac-type equation which can be 
solved almost analytically [21], see also [22]. But the resulting baryon vertex functions are then independent of the relative momentum between quark and diquark and therefore not suitable for form factor calculations.

To avoid UV divergencies in the integral equations (6) and (7) it is convenient to work with a finite extension of the interaction (quark exchange) in momentum space by modifying the exchanged quark propagator according to

$$
S(q) \rightarrow S(q) \frac{\Lambda^{2}}{q^{2}+\Lambda^{2}}
$$

While it was shown in ref. [24], that this choice leads to sensible results for the nucleon amplitudes, there is certainly the need to calculate this "form factor", which can be related to the diquark Bethe-Salpeter amplitude, from a microscopic diquark model. Work in this direction is under progress [23] (see also [19]).

Note that the color algebra for the eqs. (6,7) projected on the the color singlet has simply provided an overall factor -1 . The corresponding factor for the color octet channel is $\frac{1}{2}$. As we assume the color singlet channel interaction to be attractive the color octet one is repulsive. Thus, using confined quarks and diquarks, no states in the octet channel can be generated.

The isospin Clebsch-Gordan coefficients are given by

$$
\left(\begin{array}{cc}
1 & -\sqrt{3} \\
-\sqrt{3} & -1
\end{array}\right)
$$

and have been already absorbed in the coefficients in eqs. (6,7).

Eqs. (6.77) determine the Bethe-Salpeter vertex functions $\Phi(P, p)$ and $\Phi^{\mu}(P, p)$. They are related to the Bethe-Salpeter wave function by multiplication with the free two-particle propagator,

$$
\begin{aligned}
\Psi(P, p) & =S(P / 2+p) D(P / 2-p) \Phi(P, p), \\
\Psi^{\mu}(P, p) & =S(P / 2+p) D^{\mu \nu}(P / 2-p) \Phi^{\mu}(P, p) .
\end{aligned}
$$

\subsection{Decomposition in Dirac Algebra}

As we want to describe a nucleon with positive energy we use a corresponding projection from the very beginning i.e. we require that the Bethe-Salpeter vertex functions and wave functions are proportional to a positive energy Dirac spinor for a particle with momentum $P$ and helicity $s, u(P, s)$. For later convenience we define the following Diracmatrix-valued quantities [25]:

$$
\chi(P, p)=\sum_{s= \pm 1 / 2} \Phi(P, p) \otimes \bar{u}(P, s), \quad \chi^{\mu}(P, p)=\sum_{s= \pm 1 / 2} \Phi^{\mu}(P, p) \otimes \bar{u}(P, s)
$$




$$
\omega(P, p)=\sum_{s= \pm 1 / 2} \Psi(P, p) \otimes \bar{u}(P, s), \quad \omega^{\mu}(P, p)=\sum_{s= \pm 1 / 2} \Psi^{\mu}(P, p) \otimes \bar{u}(P, s) .
$$

These are then proportional to the positive energy projector

$$
\Lambda^{+}=\sum_{s= \pm 1 / 2} u(P, s) \otimes \bar{u}(P, s)=\frac{1}{2}(\mathbf{1}+\hat{P})
$$

where $\hat{P}^{\mu}=P^{\mu} / i M$ is the unit momentum vector parallel to the bound state momentum. Using the projector property $\left(\Lambda^{+}\right)^{2}=\Lambda^{+}$the vertex functions can be written as

$$
\begin{aligned}
\Phi(P, p) & =\chi(P, p) \Lambda^{+} u(P, s), \\
\Phi^{\mu}(P, p) & =\chi^{\mu}(P, p) \Lambda^{+} u(P, s) .
\end{aligned}
$$

The most general Dirac decomposition for the scalar function $\chi(P, p)$ involves four independent Lorentz scalar functions, e.g.

$$
\chi(P, p)=a_{1}(P, p)+a_{2}(P, p) \not P+a_{3}(P, p) p+a_{4}(P, p) \frac{1}{2}(P p p-\not p P),
$$

whereas the one for $\chi^{\mu}(P, p)$ decomposes into twelve Lorentz axialvector functions, e.g.

$$
\gamma_{5} \chi^{\mu}(P, p)=b_{1}(P, p) P^{\mu}+b_{2}(P, p) p^{\mu}+b_{3}(P, p) \gamma^{\mu}+b_{4}(P, p) P^{\mu} P p+b_{5}(P, p) p^{\mu} p+\ldots
$$

Projection onto positive energies leads to two and six independent components, respectively. It is advantageous to choose the Dirac matrices in these expansions to be eigenfunctions to $\Lambda^{+}$. In addition, the expansion coefficients are required to fulfill the Dirac equation for a particle of mass $\pm i M$. This leads to

$$
\begin{aligned}
\chi(P, p) & =S_{1}(P, p) \Lambda^{+}+S_{2}(P, p) \Xi \Lambda^{+} \\
\chi^{\mu}(P, p) & =A_{1}(P, p) \gamma_{5} \hat{P}^{\mu} \Xi \Lambda^{+}+A_{2}(P, p) \gamma_{5} \hat{P}^{\mu} \Lambda^{+} \\
& +B_{1}(P, p) \gamma_{5} \hat{p}_{T}^{\mu} \Xi \Lambda^{+}+B_{2}(P, p) \gamma_{5} \hat{p}_{T}^{\mu} \Lambda^{+} \\
& +C_{1}(P, p) \gamma_{5}\left(i\left(\hat{P}^{\mu} \mathbf{1}-\gamma^{\mu}\right)-\hat{p}_{T}^{\mu} \Xi\right) \Lambda^{+}+C_{2}(P, p) \gamma_{5}\left(i\left(\hat{P}^{\mu} \mathbf{1}+\gamma^{\mu}\right) \Xi-\hat{p}_{T}^{\mu}\right) \Lambda^{+}
\end{aligned}
$$

where

$$
\Xi=-i(p-\hat{P} \cdot p) / \sqrt{p_{T}^{\mu} p_{T}^{\mu}}
$$

is a Dirac matrix whose interpretation is obvious in the center-of-mass frame of the bound state, see below, and

$$
p_{T}^{\mu}=p^{\mu}-\hat{P}^{\mu}(\hat{P} \cdot p) \quad \text { and } \quad \hat{p}_{T}^{\mu}=p_{T}^{\mu} / \sqrt{p_{T}^{\mu} p_{T}^{\mu}}
$$

are projections of the relative momentum on the direction transverse to the total momentum, $\hat{p}_{T}^{\mu} P^{\mu}=0$. Note that the normalization applied in eq. (22) leads to easily in- 
terpretable expressions of the amplitudes in the rest frame. Nevertheless, for numerical treatment, we will modify the normalization slightly, see below.

The notation in eqs. (20), (21) is such that an index 1 refers to an operator in the expansion being a solution to a Dirac equation with mass $+i M$ (upper component in the rest frame of the bound state) and an index 2 refers to an operator in the expansion being a solution to a Dirac equation with mass $-i M$ (lower component in the rest frame of the bound state).

Let us detail this for the case of the scalar diquark. Obviously, in the rest frame of the bound state, $P=(0,0,0, i M)$, the operators read

$$
\Lambda^{+}=\left(\begin{array}{cc}
1 & 0 \\
0 & 0
\end{array}\right), \quad \Xi \Lambda^{+}=\left(\begin{array}{cc}
0 & 0 \\
\hat{\boldsymbol{p}} \boldsymbol{\sigma} & 0
\end{array}\right)
$$

Thus, multipliying the amplitude $\chi$ with an positive energy spinor $u$, using the definition

of $\chi(\sqrt{13})$ and the relation $\bar{u}(P, s) u(P, t)=\frac{E}{M} \delta_{s t}=\delta_{s t}$ (the last equal sign is only valid because we have specialized to the rest frame of the bound state) one obtains:

$$
\Phi(P, p)=\left(\begin{array}{c}
1 S_{1}(P, p) \\
\hat{\boldsymbol{p}} \boldsymbol{\sigma} S_{2}(P, p)
\end{array}\right)
$$

As anticipated this is the spinor for a $\frac{1}{2}^{+}$particle. The more complicated case of the vector spinor $\Phi^{\mu}$ relating to the amplitude with the axialvector diquark as a constituent is given in Appendix A.

\subsection{Expansion in Hyperspherical Coordinates}

In order to obtain a numerical solution of the diquark-quark Bethe-Salpeter equation we expand the amplitudes $S_{i}, A_{i}, B_{i}$ and $C_{i}, i=1,2$, in hyperspherical harmonics. The basic idea hereby is the would-be $\mathrm{O}(4)$ symmetry of the Bethe-Salpeter equation if the exchanged particle would be massless. Thus, the hope is that only a few orders are sufficient for a numerically precise solution which will be indeed the case, see [24] and the next section.

First, let us recall that in four dimensional spherical coordinates,

$$
p^{\mu}=p(\cos \phi \sin \theta \sin \psi, \sin \phi \sin \theta \sin \psi, \cos \theta \sin \psi, \cos \psi),
$$

the spherical spinor harmonics are given by

$$
\mathcal{Z}_{n j l m}(\psi, \theta, \phi)=\sqrt{\frac{2^{2 l+1}(n+1)(n-l) !(l !)^{2}}{\pi(n+l+1) !}} \sin ^{l} \psi C_{n-l}^{1+l}(\cos \psi) \mathcal{Y}_{l m}^{j}(\theta, \phi)
$$

Hereby the $C_{n-l}^{1+l}(\cos \psi)$ are the Gegenbauer polynomials [26]. The ones with $\mathrm{O}(3)$ angular 
momentum $l=0$ are identical to the Chebychev polynomials of the second kind,

$$
C_{n}^{1}(\cos \psi)=T_{n}(\cos \psi)=\frac{\sin (n+1) \psi}{\sin \psi}
$$

obeying the orthogonality relation

$$
\int_{0}^{\pi} d \psi \sin ^{2} \psi T_{n}(\cos \psi) T_{m}(\cos \psi)=\frac{\pi}{2} \delta_{n m}
$$

The $\mathrm{O}(3)$ spherical spinor harmonics $\mathcal{Y}_{l m}^{j}(\theta, \phi)$ are the usual ones familiar from relativistic quantum mechanics. They obey the relation

$$
\mathcal{Y}_{j \pm 1 / 2, m}^{j}(\hat{p})=-\hat{\boldsymbol{p}} \boldsymbol{\sigma} \mathcal{Y}_{j \mp 1 / 2, m}^{j}(\hat{p}) .
$$

Using the usual spherical harmonics $Y_{l m}$ the explicit expression is given by

$$
\mathcal{Y}_{l m}^{j}(\theta, \phi)=\frac{1}{\sqrt{2 l+1}}\left(\begin{array}{c} 
\pm \sqrt{l \pm m+\frac{1}{2}} Y_{l, m-1 / 2} \\
\sqrt{l \mp m+\frac{1}{2}} Y_{l, m+1 / 2}
\end{array}\right)
$$

for $j=l+\frac{1}{2}$ and $j=l-\frac{1}{2}, l>0$, respectively.

The amplitudes in eqs. (20), (21) $S_{i}, A_{i}, B_{i}$ and $C_{i}, i=1,2$, are expanded in Chebychev polynomials, e.g.

$$
S_{i}(P, p)=\sum_{n=0}^{\infty} i^{n} S_{i n}\left(P^{2}, p^{2}\right) T_{n}(\hat{P} \cdot \hat{p})
$$

and analogous for the functions $A_{i}, B_{i}$ and $C_{i}$.

\subsection{Numerical method}

For the numerical solution of the Bethe-Salpeter equation we will restrict ourselves in the following to $0^{+}$diquarks, i.e the solution of eq. (6) with $g_{a}=0$. The inclusion of $1^{+}$diquarks and accordingly the axialvector amplitudes of the nucleon is much more involved and will be treated in a forthcoming publication. Furthermore we choose equal masses for the quark and the diquark, $m_{q} \equiv m_{s}$. Then all dimensional quantities in the Bethe-Salpeter equation can be expressed in units of the constituent mass $m_{q}$. To allow a comparison of our results with the ones reported in [24] we also choose, if not stated otherwise, $\Lambda=2 m_{q}$, which basically fixes the width of the interaction in momentum space.

When working in the rest frame of the bound state we parametrize the relative momenta in eq. (6) according to

$$
p_{\mu}^{\prime}=p^{\prime}\left(\cos \phi^{\prime} \sin \theta^{\prime} \sin \psi^{\prime}, \sin \phi^{\prime} \sin \theta^{\prime} \sin \psi^{\prime}, \cos \theta^{\prime} \sin \psi^{\prime}, \cos \psi^{\prime}\right),
$$




$$
p_{\mu}=p(0,0, \sin \psi, \cos \psi)
$$

By changing the normalization for the Dirac matrix in $(22)$ to

$$
\tilde{\Xi}=-i(p-\hat{P} \cdot p) / \sqrt{p^{\mu} p^{\mu}}
$$

and denoting the spatial part of the normalized 4-vector $p$ by $\tilde{\boldsymbol{p}}$, the generic structure of the integral equation can be written as

$$
\begin{gathered}
\left(\begin{array}{cc}
\mathbf{1} S_{1}(P, p) & 0 \\
\sin \psi \sigma_{3} S_{2}(P, p) & 0
\end{array}\right)=-g_{s}^{2} \int \frac{d^{4} p^{\prime}}{(2 \pi)^{4}} K\left(p, p^{\prime}, P\right)\left(\begin{array}{cc}
\mathbf{1} S_{1}\left(P, p^{\prime}\right) & 0 \\
\tilde{\boldsymbol{p}}^{\prime} \boldsymbol{\sigma} S_{2}\left(P, p^{\prime}\right) & 0
\end{array}\right) \\
K\left(p, p^{\prime}, P\right) \sim \gamma_{5} S(-q) \gamma_{5} S\left(p_{a}^{\prime}\right) D\left(p_{b}^{\prime}\right)
\end{gathered}
$$

Using the expansion of the amplitudes in terms of Gegenbauer polynomials (31) and applying the orthogonality relation (28), we extract the expansion functions (amplitudes) $S_{i, m}(p),(i=1,2 ; m=0 . . \infty)$ and obtain after integration over $\phi^{\prime}$

$$
\left(\begin{array}{c}
S_{1 m}(p) \\
S_{2 m}(p)
\end{array}\right)=g_{s}^{2} \sum_{n=0}^{\infty} i^{n-m} \int d \Sigma \int_{0}^{\infty} d p^{\prime} p^{\prime}\left(\begin{array}{cc}
\tilde{K}_{11} & \tilde{K}_{12} \\
\tilde{K}_{21} & \tilde{K}_{22}
\end{array}\right) \cdot\left(\begin{array}{c}
S_{1 n}\left(p^{\prime}\right) \\
S_{2 n}\left(p^{\prime}\right)
\end{array}\right)
$$

By taking the spin degeneracy into account we reduced the problem to two coupled integral equations, where $\tilde{K}_{i, j},(i, j=1,2)$ now denote real scalar quantities and can be calculated straightforwardly. The remaining angular integrals are given by

$$
\int d \Sigma=\int_{0}^{\pi} d \psi \sin ^{2} \psi \int_{0}^{\pi} d \psi^{\prime} \sin ^{2} \psi^{\prime} \int_{0}^{\pi} d \Theta^{\prime} \sin \Theta^{\prime}
$$

For the actual solution the Gegenbauer expansion has to be terminated at a finite $n=$ $m_{\max }$ and due to the structure of eq. (37) $m$ is in the range $0 \leq m \leq m_{\max }$. Equation (37) is now the most convenient expression of the Bethe-Salpeter equation for numerical treatment. While the $\Theta^{\prime}$ integration can be done analytically, we perform the $\psi$ and $\psi^{\prime}$ integration numerically by means of Gaussian quadratures with appropriate weights $\left(w(z)=\sqrt{1-z^{2}}, z=\cos \psi\right)$. The final step is then to discretize $p$ and $p^{\prime}$ with $k_{\text {max }}$ grid points to generate a $k_{\max } \times k_{\max }$ mesh in momentum space. Actually we map the interval $[0, \infty]$ to $[-1,1]$ and determine the grid points according to a Gaussian quadrature with Gauss-Legendre weights. In this way a $\left(2 \cdot m_{\max } \cdot k_{\max } \times 2 \cdot m_{\max } \cdot k_{\max }\right)$ matrix is generated which can be treated as an eigenvalue problem; for a given bound state mass $M$ (which appears nonlinearly in the integral equation) we seek the corresponding coupling constant $g_{s}$. In order to test the numerics we solved the eigenvalue problem by straightforward diagonalization as well as by an iteration method and found, within numerical accuracy, identical results. When applying the iteration method one starts with initial guesses for the amplitudes and iterates the matrix equation until $g_{s}$ converges.

As a further check of our numerical procedure we also solved the Bethe-Salpeter equation 
involving explicitly the wavefunction $\Psi(P, p)$ by iteration,

$$
\begin{aligned}
& \Phi(P, p)=-g_{s}^{2} \int \frac{d^{4} p^{\prime}}{(2 \pi)^{4}} H\left(p, p^{\prime}\right) \Psi\left(P, p^{\prime}\right) \\
& \Psi(P, p)=D(P / 2-p) S(P / 2+p) \Phi(P, p) .
\end{aligned}
$$

Note that the corresponding kernel $H \sim \gamma_{5} S(-q) \gamma_{5}$ is independent of $P$, or $M$, which in principle allows a fast determination of $M$ with given $g_{s}$. First we use an adequate decomposition for $\Psi(P, p)$ as in eq. (25),

$$
\Psi(P, p)=\left(\begin{array}{c}
1 G_{1}(P, p) \\
\sin \psi \sigma_{3} G_{2}(P, p)
\end{array}\right) .
$$

We then also expand the free two-particle propagator $D(P / 2-p) S(P / 2+p)$ in terms of Gegenbauer polynomials (obtained in the case of tree-level propagators analytically, in the case of confining propagators numerically). This defines the coefficients $u_{i j}$ via the relation

$$
\begin{array}{r}
D(P, p) S(P, p)\left(\begin{array}{c}
1 S_{1}(P, p) \\
\sigma_{3} \sin \psi S_{2}(P, p)
\end{array}\right)=\sum_{k_{1}=0}^{\infty} i^{k_{1}}\left(\begin{array}{cc}
u_{11}^{k_{1}} & u_{12}^{k_{1}} \\
\sigma_{3} \sin \psi u_{21}^{k_{1}} & \sigma_{3} \sin \psi u_{22}^{k_{1}}
\end{array}\right) \times \\
T_{k_{1}}(\cos \psi)\left(\begin{array}{c}
S_{1}(P, p) \\
S_{2}(P, p)
\end{array}\right) .
\end{array}
$$

The final step is the projection onto the expansion coefficients $S_{i m}(p)$ and $G_{i m}(p), i=1,2$, to yield

$$
\begin{gathered}
\left(\begin{array}{l}
S_{1 m}(p) \\
S_{2 m}(p)
\end{array}\right)=g_{s}^{2} \sum_{n=0}^{n_{\max }} i^{n-m} \int d \Sigma \int_{0}^{\infty} d\left|p^{\prime}\right|\left|p^{\prime}\right|^{3}\left(\begin{array}{ll}
\tilde{H}_{11}^{\prime} & \tilde{H}_{12}^{\prime} \\
\tilde{H}_{21}^{\prime} & \tilde{H}_{22}^{\prime}
\end{array}\right) \cdot\left(\begin{array}{l}
G_{1 n}\left(p^{\prime}\right) \\
G_{2 n}(p)
\end{array}\right) \\
\left(\begin{array}{c}
G_{1 n}(p) \\
G_{2 n}(p)
\end{array}\right)=\sum_{m=0}^{m_{\max }} \sum_{k_{1}=0}^{m+n_{\max }} i^{k_{1}+m-n}\left(\begin{array}{cc}
u_{11}^{k_{1}} & u_{12}^{k_{1}} \\
u_{21}^{k_{1}} & u_{22}^{k_{1}}
\end{array}\right) \cdot\left(\begin{array}{c}
S_{1 m}(p) \\
S_{2 m}(p)
\end{array}\right) \\
\left|k_{1}-m\right| \leq n \leq k_{1}+m \\
k_{1}+m \equiv n \bmod 2
\end{gathered}
$$

which is suitable for an iteration procedure. Note, that we used the following addition theorem for Gegenbauer polynomials:

$$
T_{n}(z) T_{m}(z)=T_{|n-m|}(z)+T_{|n-m|+2}(z)+\ldots+T_{n+m}(z) .
$$

Once the kernel $H\left(p, p^{\prime}\right)$ was computed on a momentum mesh we iterated $\Phi(P, p)$ (expanded up to order $\left.m_{\max }\right)$ taking into account $\Psi(P, p)$ up to order $n_{\max }$, as expressed in (43).

In the limit $n_{\max } \rightarrow \infty$ both methods are by construction equivalent. In the actual calculations $n_{\max }=m_{\max }+3$ proved to be sufficient as the eigenvalues for a given $m_{\max }$ 
quickly converged due to the asymptotic behaviour of the kernel for higher orders $m$ and $n$.

Before discussing our numerical results we want to mention that the linear Bethe-Salpeter equation provides no a priori normalization condition. While a physical normalization of the bound state amplitudes will be discussed in the next section here we simply demand $S_{10}\left(p_{1}\right)=1$ ( $p_{1}$ denotes the first grid point of the momentum mesh), which provides a preliminary overall normalization.

\subsection{Numerical results}

In this subsection we discuss our numerical results. By examining the convergence properties of the integral equation we observed that for $k_{\max } \geq 30$ the eigenvalues and eigenvectors do not depend any more on the size of the momentum mesh. The numerically obtained amplitudes are shown in figure 2. Type I denotes the calculations where quark and diquark propagators with poles are included ( $c f . f \equiv 1$ in eq. (2)); type II denotes calculations with confining propagators. When doing the calculation with propagators of type II we also vary $d$, the damping factor in the exponentials. As it is seen in the figures if $d$ is increased, the amplitudes approach the results obtained with type I propagators. For the case $d=10$ there is no visible deviation from the type I amplitudes. This feature is expected since for a large damping factor basically only the timelike properties of the quark and diquark propagators are affected by the exponential. We note that the amplitudes for the type I calculations are in agreement with the results in [24], which provides a check of our calculation.

Table 1

\begin{tabular}{||c||c|c|c||}
\hline \multicolumn{3}{||c||}{ type $\mathrm{I}$} \\
\hline$m_{\max }$ & $\mathrm{M}=m_{q}$ & $\mathrm{M}=1.9 m_{q}$ & $\mathrm{M}=1.99 m_{q}$ \\
\hline 0 & 15.8164 & 9.9237 & 8.2132 \\
1 & 16.1141 & 9.8853 & 8.1729 \\
2 & 16.0809 & 9.8488 & 8.1496 \\
3 & 16.0812 & 9.8485 & 8.1494 \\
\hline
\end{tabular}

Eigenvalues of the Bethe-Salpeter equation with type I propagators for different nucleon masses when the expansion in terms of Gegenbauer polynomials is terminated at $m=m_{\max }$.

From figure (2) it can now be seen that the employed expansion in terms of Gegenbauer polynomials converge rapidly: The absolute value of subsequent amplitudes (compare $e . g$. $S_{11}$ with $\left.S_{10}\right)$ decreases roughly by one order of magnitude. So higher orders $(n \geq 3)$ can be safely neglected. 
$S_{10}(p)$

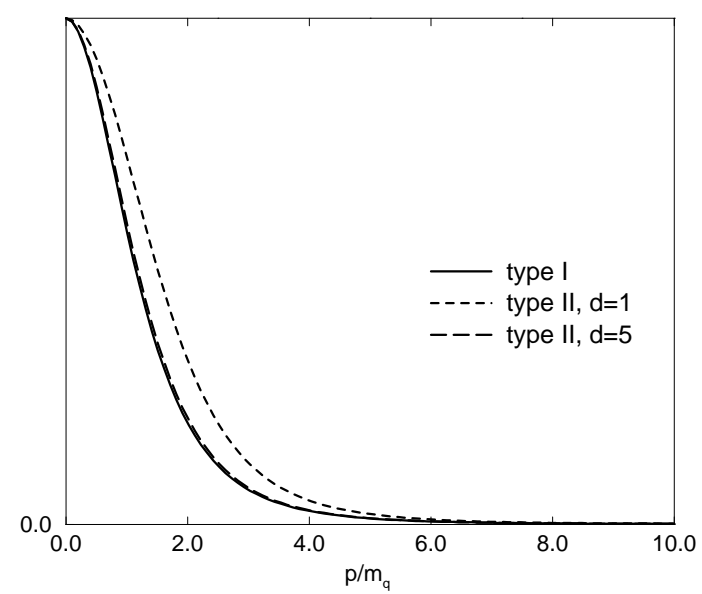

$S_{11}(p)$

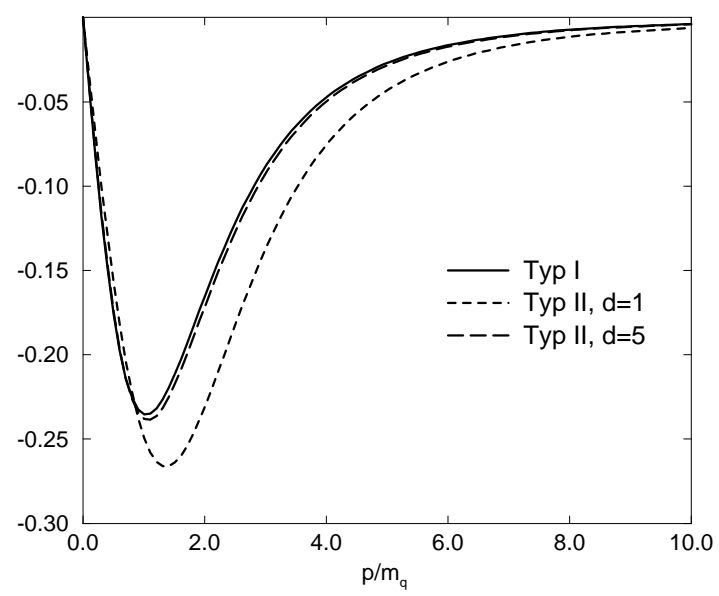

$S_{12}(p)$

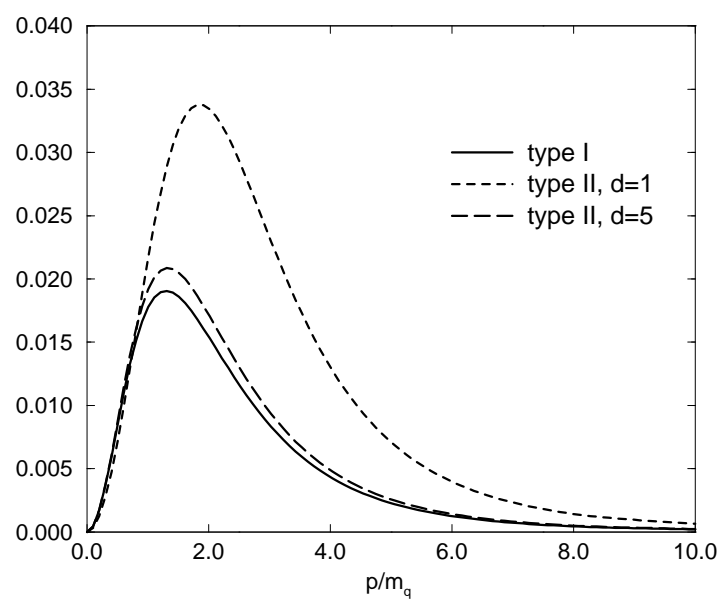

$S_{20}(p) / p$

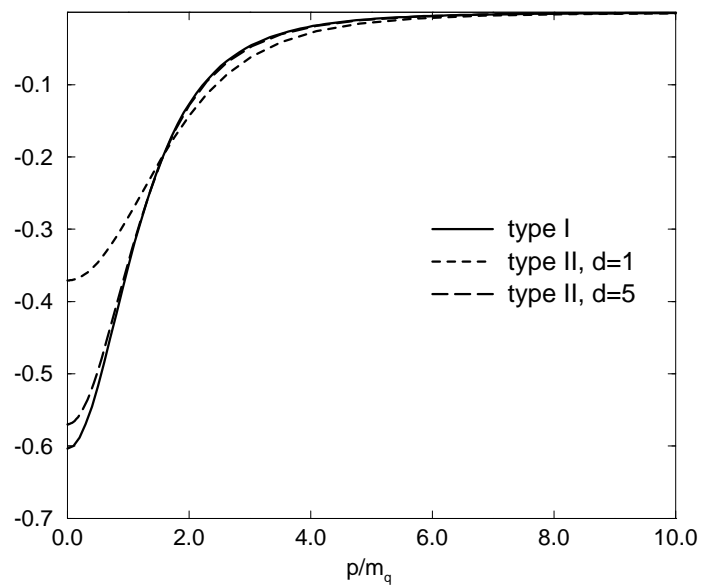

$S_{21}(p) / p$

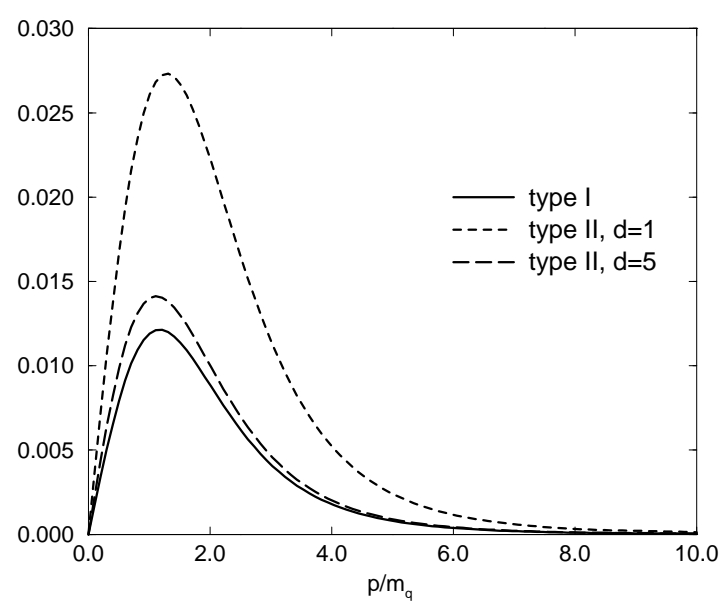

$\mathrm{S}_{22}(\mathrm{p}) / \mathrm{p}$

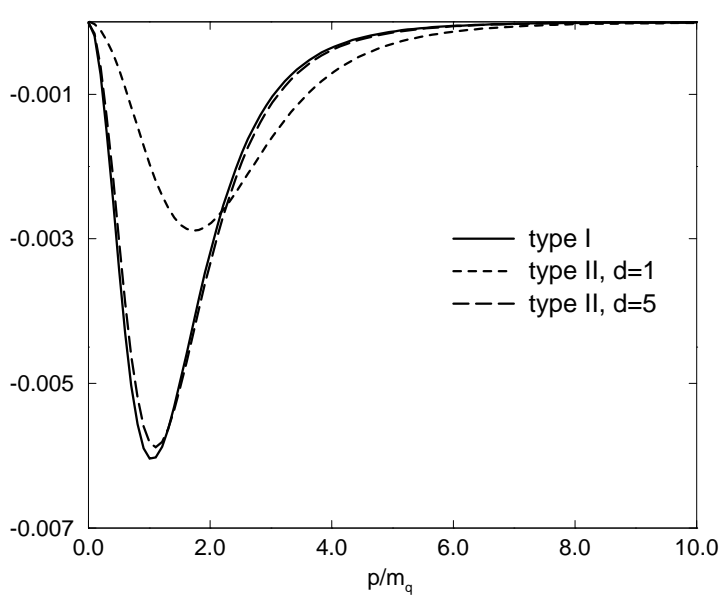

Fig. 2. Bethe-Salpeter amplitudes $S_{10}(p) \ldots S_{22}(p)$ calculated with type I and type II propagators (with different values for $d$ ) and with $k_{\max }=30$. 
Table 2

\begin{tabular}{||c||c|c|c||}
\hline \multicolumn{4}{||c||}{ type II, $\mathrm{d}=1$} \\
\hline$m_{\max }$ & $\mathrm{M}=m_{q}$ & $\mathrm{M}=1.9 m_{q}$ & $\mathrm{M}=1.99 m_{q}$ \\
\hline 0 & 17.7627 & 14.0058 & 13.5747 \\
1 & 18.2856 & 14.0938 & 13.6373 \\
2 & 18.2502 & 14.0485 & 13.5916 \\
3 & 18.2507 & 14.0487 & 13.5918 \\
\hline
\end{tabular}

Eigenvalues of the Bethe-Salpeter equation with type II and $d=1$ propagators for different bound state masses when the expansion in terms of Gegenbauer polynomials is terminated at $m=m_{\max }$.

In order to stress this fact, we show in table (11) and (2) how the eigenvalues of the integral equation converge when more orders of the Gegenbauer expansion are included in the calculation, i.e. $m_{\max }$ is increased. The same behaviour can be observed when the eigenvectors (amplitudes) are examined; graphically they then become undistinguishable. Thus, we conclude that especially in the "physical region" of the binding energy $\left(m_{q} \leq\right.$ $M \leq 1.9 m_{q}$ ) sufficient convergence is achieved with $n_{\max }=2$, thereby including 3 orders in the expansion.

Though the two methods to solve the Bethe-Salpeter equation (one for the nucleon vertex function and one for the wave function) mentioned in the last section are expected to give equivalent results, deviations might arise in the numerical integration procedure. The kernel $K\left(p, p^{\prime} ; P\right)$, including the free two-particle propagator approaches zero much faster than $H\left(p, p^{\prime}\right)$ for large values of $p^{\prime}$ and for higher orders $m, n$ in the Gegenbauer expansion. Therefore, using the second method, the number of grid points has to be increased to obtain the same numerical accuracy; we typically chose 50 points for the momentum mesh. Then the results agree very well within numerical accuracy.

Having in mind that $S_{2}(p)$ describes the lower component of the nucleon Dirac field (which is usually neglected, e.g. in nonrelativistic calculations), it is surprising that we obtain rather large "small" components. This was also observed in ref. [24]. To study this in greater detail we plot in figure (3) the amplitude $S_{20}(p)$ for different values of the bound state mass. For both types of propagators the maximum of the amplitudes $S_{20}(p)$ increases when the binding energy $E_{B}=2 m_{q}-M$ tends to 0 . Note however that this result depends on the chosen representation of the fermion field (in our case the Dirac representation) We will investigate in the next section whether the large lower component of the nucleon spinor has effects on observables where a $\gamma_{5}$ is involved in the coupling to the external current $\left(g_{A}\left(Q^{2}\right)\right.$ and $\left.g_{\pi N N}\left(Q^{2}\right)\right)$ because this $\gamma$-matrix induces a mixing between upper and lower components which give rise to contributions to these observables.

The dependence of the amplitudes on the width of the interaction can be seen from fig. (4). Although we restricted ourselves in the plot to a calculation of $S_{10}(p)$ for type II

$\overline{5}$ We thank F. Lenz for this remark. 
$\mathrm{S}_{20}(\mathrm{p}) / \mathrm{p}$, type I

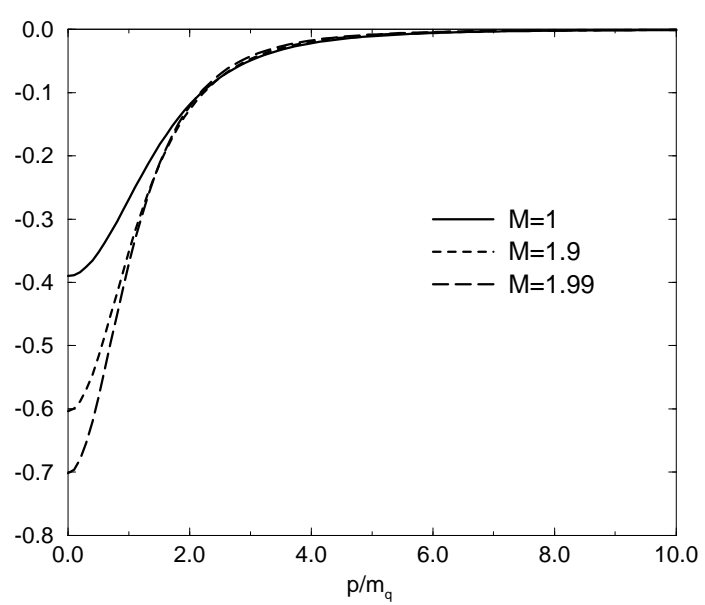

$\mathrm{S}_{20}(\mathrm{p}) / \mathrm{p}$, type II

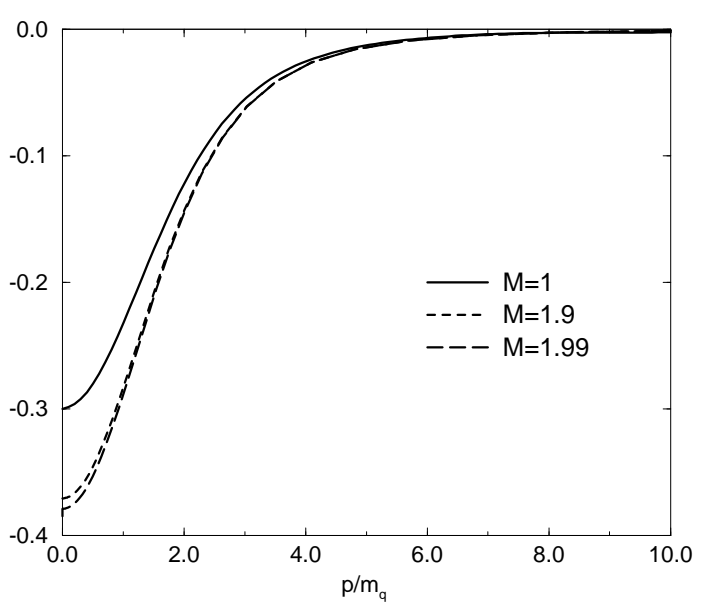

Fig. 3. The lower component $S_{20}(p)$ for $M=m_{q}, 1.9 m_{q}, 1.99 m_{q}$ using type I propagators, left hand figure, and type II propagators with $d=1$, right hand figure.

$$
\mathrm{S}_{10}(\mathrm{p})
$$

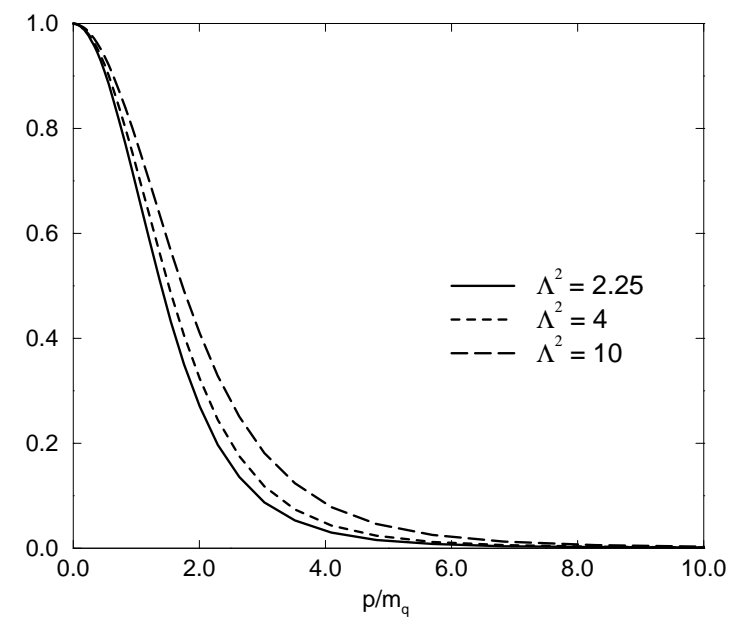

Fig. 4. $S_{10}(p)$ is plotted for type II and $d=1$ propagators for different values of $\Lambda^{2}$ (in units of $m_{q}^{2}$ ) which controls the range of the interaction.

propagators with $d=1$ and a bound state mass of $M=1.9 m_{q}$ the observed behaviour is quite generic: When $\Lambda$ is increased, higher modes of the interaction are included, leading to a broader amplitude in momentum space. Correspondingly, in coordinate space the interaction become shorter ranged leading to a narrower amplitude. Although this behaviour would allow to fit $\Lambda$ e.g. to the mean square radius of the nucleon, we will not do this in our following calculation.

In order to demonstrate the main advantage of our approach, namely working not only with free (type I) propagators but also with confining propagators (type II), we display in figure 5 the calculated coupling constant $g_{s}$ ( eigenvalue) as a function of the nucleon mass. For type I propagators the eigenvalues near threshold start to decrease rapidly. Ideally they should display an infinite slope for $M=2 m_{q}$, because there the bound 
Eigenvalue vs. Bound State Mass

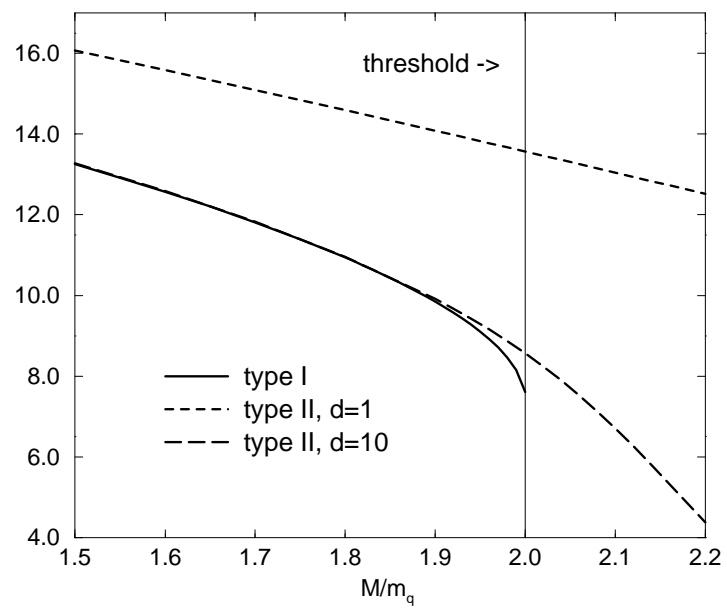

Fig. 5. Eigenvalues of the integral equation as functions of the bound state mass $M$. In case of type I propagators, the influence of the quark-diquark threshold is clearly visible.

state decays into its constituents. To observe this feature a much more refined numerical method (suitable for extremely loose bound states) would be necessary. Our numerical method, which is very robust in a wide range of bound state masses cannot account for this property, but signals the nearby threshold by a strong sensitivity of $g_{s}$ on $M$. When using now the confining propagators we get a totally different behaviour: The eigenvalues are not affected by the "threshold" any more (which in this case is no threshold at all). The function $g_{s}$ is a smooth function and nothing signals the possibility of the decay of the bound state. In this way confinement is realized in our approach: By choosing confining propagators for the constituents of the nucleon, we exclude its unphysical decay into free quarks and diquarks. Even when the damping factor $d$ is increased, so that for $M \leq 1.9$ the eigenvalues and eigenvectors of a type I calculation are basically recovered, near "threshold" we again observe confinement.

Before we describe in the next section the application of the so far obtained amplitudes in a calculation of baryonic matrix elements, we want to mention that the numerical solutions of the Bethe-Salpeter equation can be parametrized for further applications very effectively. By examining the infrared and ultraviolet asymptotics of the amplitudes we find simple rational functions with parameters fitted to the numerical solutions. For details we refer to appendix $B$.

\section{Nucleon Form Factors}

In order to test the validity of the proposed quark-diquark picture of the nucleon, it is not enough just to solve the corresponding Bethe-Salpeter equation, since its solution, the Bethe-Salpeter amplitudes (or the vertex functions) have no physical interpretation by themselves. It is therefore necessary to use the solution of the Bethe-Salpeter equation to calculate physical observables as, e.g. form factors. Especially we are interested in electromagnetic form factors of proton and neutron, the pion-nucleon form factor $g_{\pi N N}$ 
and the axial form factor $g_{A}$. These observables are accessible by coupling an appropriate probing external current to the nucleon.

In ref. 28.29] a comparable calculation to our approach was performed in the context of the NJL-model (with unconfined quarks and diquarks), but only static properties (vanishing momentum transfer) of the nucleon were considered; in ref. [30 spacelike form factors were calulated (also within the NJL model) but assuming a static quark exchange between quark and diquark and therefore working with a "trivial" nucleon vertex function. A calculation within the Salpeter approach, instead of the here employed fully relativistic Bethe-Salpter equation, is reported in ref. [31] and [32]. So far, there exist no work in which a) the BS equation was solved without any approximation for the quark exchange and $\mathrm{b}$ ) the resulting solution was used to evaluate observables for finite momentum transfer to the nucleon. See, however, ref. 24] for a calculation of nucleon structure functions within this approach.

At this point we make the following remarks: The so far developed picture of a nucleon consisting of a quark and a scalar diquark with equal masses is certainly to naïve for a correct descripton of the nucleon and therefore quantitatively agreement with experimental results cannot be expected. Nevertheless we believe that the reported studies are a necessary step in the development of a complete baryon model. Therefore we not even try to fit e.g. $\Lambda$ or $d$ to experimental numbers. Our aim is rather to observe, whether certain nucleon properties can be described reasonably.

The calculation of hadronic matrix elements between bound state vertex functions is conveniently performed in Mandelstam's formalism [33], which will be introduced in the next subsection.

\subsection{Mandelstam's Formalism}

In order to calculate the matrix element of an external current operator (generically denoted by $\hat{O}$ ) between bound state amplitudes we use Mandelstam's formalism 33 (see also [29]), which reads in momentum space

$$
\begin{aligned}
\left\langle\hat{O}_{(\mu)}\right\rangle & =\left\langle N\left(P_{f}, S_{f}\right)\left|\hat{O}_{(\mu)}\right| N\left(P_{i}, S_{i}\right)\right\rangle=\frac{1}{\sqrt{4 E_{P_{f}} E_{P_{i}}}} J_{(\mu)}\left(P_{f}, P_{i}\right), \\
J_{(\mu)}\left(P_{f}, P_{i}\right) & =\int \frac{d^{4} p_{f}}{(2 \pi)^{4}} \int \frac{d^{4} p_{i}}{(2 \pi)^{4}} \bar{\Psi}\left(P_{f}, p_{f}\right) \Gamma_{\hat{O}}\left(p_{f}, P_{f} ; p_{i}, P_{i}\right) \Psi\left(P_{i}, p_{i}\right) .
\end{aligned}
$$

Here $P_{i}, p_{i}$ and $P_{f}=P_{i}+Q, p_{f}$ denote the total and the relative momenta of the quarkdiquark bound state before and after the interaction with the external current, while $\Psi$ and $\bar{\Psi}$ are the properly normalized Bethe-Salpeter wave functions. By writing explicitly the quark and diquark propagators, which are included in the definition of the BS-wave function, we obtain Mandelstam's prescription in a representation, which contains the nucleon vertex function, calculated in the previous section.

$$
\begin{array}{r}
J_{(\mu)}\left(P_{f}, P_{i}\right)=\int \frac{d^{4} p_{f}}{(2 \pi)^{4}} \int \frac{d^{4} p_{i}}{(2 \pi)^{4}} \bar{\chi}\left(P_{f}, p_{f}\right) D\left(\frac{1}{2} P_{f}-p_{f}\right) S\left(\frac{1}{2} P_{f}+p_{f}\right) \times \\
\Gamma_{\hat{O}}\left(p_{f}, P_{f} ; p_{i}, P_{i}\right) D\left(\frac{1}{2} P_{i}-p_{i}\right) S\left(\frac{1}{2} P_{i}+p_{i}\right) \chi\left(P_{i}, p_{i}\right) .
\end{array}
$$




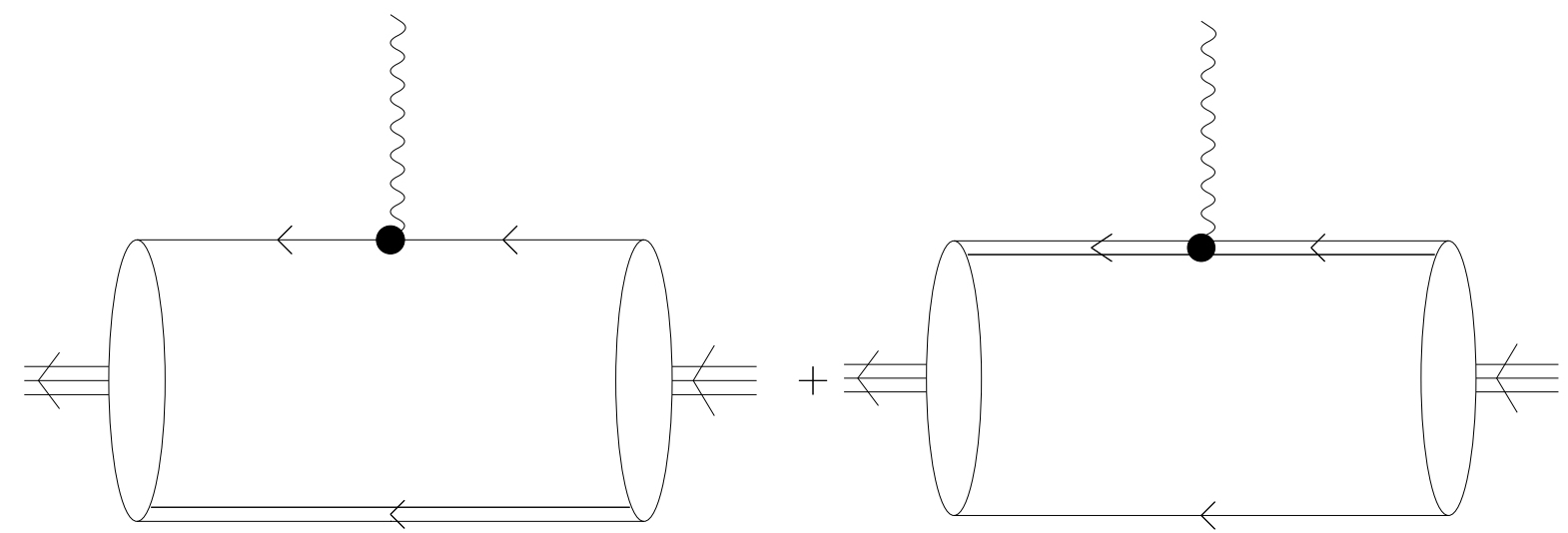

Fig. 6. The two diagrams which contribute to the nucleon matrix elements in impulse approximation.

This representation allows the direct evaluation of the nucleon current. The 5-point function $\Gamma_{\hat{O}}$ appearing in eq. (47) and (48) describes the coupling of the probing current with momentum $Q$ to the constituents of the bound state.

In the following we work in a generalized impulse approximation, where the coupling to the diquark and the constituent quark is considered, while we neglect, in this first calculation, the coupling to the exchanged quark.

Accordingly the 5-point function is defined by

$$
\begin{aligned}
\Gamma_{\hat{O}}\left(p_{f}, P_{f} ; p_{i}, P_{i}\right) & =\delta\left(p_{f}-p_{i}-\frac{1}{2} Q\right) D^{-1}\left(\frac{1}{2} P_{i}-p_{i}\right) \Gamma_{\hat{O}}^{q}\left(p_{f}, P_{f} ; p_{i}, P_{i}\right) \\
& +\delta\left(p_{f}-p_{i}+\frac{1}{2} Q\right) S^{-1}\left(\frac{1}{2} P_{i}+p_{i}\right) \Gamma_{\hat{O}}^{d}\left(p_{f}, P_{f} ; p_{i}, P_{i}\right)
\end{aligned}
$$

Inserting $\Gamma_{\hat{O}}$ into eq. (48), one observes that the matrix element is split into a quark and a diquark part

$$
J_{(\mu)}\left(Q^{2}\right)=J_{(\mu)}^{q}\left(Q^{2}\right)+J_{(\mu)}^{d}\left(Q^{2}\right) .
$$

If the external nucleon legs are on their mass shells $\left(P_{i}^{2}=P_{f}^{2}=-M^{2}\right)$, the nucleon current depends only on the squared momentum $Q^{2}$ of the probing external current. The two terms appearing in (50) are given by the loop integrals

$$
J_{(\mu)}^{q}\left(Q^{2}\right)=\int \frac{d^{4} p}{(2 \pi)^{4}} \bar{\chi}\left(P_{f}, p_{f}\right) S\left(p_{+}\right) \Gamma_{(\mu)}^{q}\left(p_{+}, p_{-}\right) S\left(p_{-}\right) D\left(p_{d}\right) \chi\left(P_{i}, p_{i}\right)
$$

and

$$
J_{(\mu)}^{d}\left(Q^{2}\right)=\int \frac{d^{4} p}{(2 \pi)^{4}} \bar{\chi}\left(P_{f}, p_{f}\right) D\left(p_{+}\right) \Gamma_{(\mu)}^{d}\left(p_{+}, p_{-}\right) D\left(p_{-}\right) S\left(p_{q}\right) \chi\left(P_{i}, p_{i}\right) .
$$

In appendix $\square$ we discuss the momentum rooting in the loop integrals and their numerical evaluation.

Since the Bethe-Salpeter equation does not provide the overall normalization of the bound state vertex function, a physical normalization condition has to be imposed. The usual requirement, a unit residue of the bound state propagator at the mass pole [34], which in 
our formalism reads

$$
\int \frac{d^{4} p}{(2 \pi)^{4}} \bar{\chi}(P, p)\left[\frac{\partial}{\partial P_{\mu}} S\left(\frac{1}{2} P+p\right) D\left(\frac{1}{2} P-p\right)\right] \chi(P, p)=2 \Lambda^{+} P^{\mu}
$$

then leads to the correct normalization. Since the quark exchange is independent of the c.m. momentum $P$, it does not contribute to the normalization of the vertex function. Note that the adjoint vertex function $\bar{\chi}(P, p)$ has to fulfill

$$
\bar{\chi}(P, p)=\Lambda^{+} \bar{\chi}(P, p)
$$

and is therefore given by $\bar{\chi}(P, p)=\gamma_{4} \chi^{\dagger}(P, p) \gamma_{4}$.

\subsection{Electromagnetic Form Factors}

To determine the electromagnetic (e.m.) form factors one has to calculate the e.m. nucleon current. The probing current is an external photon and therefore the quark-photon and the diquark-photon vertex functions are needed to apply Mandelstam's formalism. Electromagnetic gauge invariance is manifest in the Ward-Takahashi identity,

$$
Q_{\mu} \Gamma_{\mu}(p+Q, p)=S^{-1}(p+Q)-S^{-1}(p),
$$

which provides the connection between the longitudinal part of the (di-)quark-photon vertexfunction and the inverse (di-)quark propagator, and for the limit $Q^{2} \rightarrow 0$ in the Ward identity

$$
\Gamma_{\mu}(p, p)=\frac{\partial}{\partial p_{\mu}} S^{-1}(p)
$$

Using vertex functions in accordance with these identities means to respect gauge invariance at the constituent level.

A vertex function which solves the above identites for the quark-photon coupling is the Ball-Chiu vertex [35, 36],

$$
\begin{aligned}
\left(\Gamma_{\mu}^{q}\right)_{e . m .}(p, k) & =\Gamma_{\mu}^{B C}(p, k)=i \frac{A\left(p^{2}\right)+A\left(k^{2}\right)}{2} \gamma_{\mu} \\
& +i \frac{(p+k)_{\mu}}{p^{2}-k^{2}}\left[\left(A\left(p^{2}\right)-A\left(k^{2}\right)\right) \frac{\gamma p+\gamma k}{2}-i\left(B\left(p^{2}\right)-B\left(k^{2}\right)\right)\right]
\end{aligned}
$$

which is determined by the quark self-energy functions $A\left(p^{2}\right)$ and $B\left(p^{2}\right)$ appearing in the quark propagator

$$
S(p)=-i \gamma \cdot p \sigma_{v}\left(p^{2}\right)+\sigma_{s}\left(p^{2}\right)=\left[i \gamma \cdot p A\left(p^{2}\right)+B\left(p^{2}\right)\right]^{-1}
$$

and can be read off from eq. (3). The vertex function (57) not only satisfies eqs. (55) and (56), but is also free of kinematical singularities, has the same properties under P,C,T transformations as the perturbative vertex $i \gamma_{\mu}$ and reduces itself to this vertex in case of 
bare quarks (this is the case when a type I quark propagator is used). In the following we will restrict ourselves to the pure longitudinal Ball-Chiu vertex and will not consider any transversal part, which cannot be determined using e.m. gauge invariance $\square$.

The vertex function of a nonperturbative (extended) scalar particle, also has to be chosen to fulfill eqs. (55) and (56); this is discussed in ref. [38]. Again we will use the simplest longitudinal diquark-photon vertexfunction

$$
\left(\Gamma_{\mu}^{d}\right)_{e . m .}(p, k)=-(p+k)_{\mu}\left[\frac{p^{2} C\left(p^{2}\right)-k^{2} C\left(k^{2}\right)}{p^{2}-k^{2}}-m_{s} \frac{C\left(p^{2}\right)-C\left(k^{2}\right)}{p^{2}-k^{2}}\right]
$$

which is determined by the self-energy function $C\left(p^{2}\right)$ of the diquark propagator

$$
D(p)=-\frac{F\left(p^{2}\right)}{p^{2}+m_{s}^{2}}=-\left(\left(p^{2}+m_{s}^{2}\right) C\left(p^{2}\right)\right)^{-1} .
$$

The explicit expression can be easily obtained when comparing with eq. (4). In particular, any transversal part of the vertex function, which would describe an anomalous magnetic moment of the scalar diquark will not be considered.

To calculate the electromagnetic form factors of the nucleon we insert

$$
\Gamma_{\mu}^{q}\left(p_{+}, p_{-}\right)=Q_{q}\left(\Gamma_{\mu}^{q}\right)_{e . m .}\left(p_{+}, p_{-}\right)
$$

and

$$
\Gamma_{\mu}^{d}\left(p_{+}, p_{-}\right)=Q_{d}\left(\Gamma_{\mu}^{d}\right)_{e . m .}\left(p_{+}, p_{-}\right)
$$

with the charge matrices in isospin space given by

$$
Q_{q}=\frac{1}{2}\left(\frac{1}{3} \mathbf{1}+\tau_{z}\right), \quad Q_{d}=\frac{1}{3}
$$

into eqs. (51) and (52), respectively, and evaluate the loop integrals. As it is well known, using Lorentz invariance, invariance under P,C,T and the fact that the incoming and outgoing nucleons are onshell, the longitudinal e.m. nucleon current can be decomposed into

$$
J_{\mu}^{e . m .}\left(Q^{2}\right)=\Lambda^{+}\left(P_{f}, S_{f}\right)\left[i M_{B}\left(F_{e}\left(Q^{2}\right)-F_{m}\left(Q^{2}\right)\right) \frac{P_{\mu}}{P^{2}}+F_{m}\left(Q^{2}\right) \gamma_{\mu}\right] \Lambda^{+}\left(P_{i}, S_{i}\right) .
$$

Note that within our formalism the Dirac spinors usually appearing on the right hand side of this equation are replaced by the $\Lambda^{+}$projectors. The Lorentz invariant functions $F_{e}\left(Q^{2}\right)$ and $F_{m}\left(Q^{2}\right)$ denote the electric and magnetic form factor, which can be extracted by taking appropriate traces. Here and in the following subsections the calculations are most conveniently performed in the Breit-frame (see appendix C).

\footnotetext{
${ }^{6}$ In ref. [37] it was shown that the requirement of multiplicative renormalisibility constrains the construction of possible transversal parts of the e.m. vertexfunction.
} 


\subsection{Pion Nucleon Form Factor}

The pion nucleon form factor, which enters into pure hadronic models usually as an input parameter, has been much debated in the last few years. Especially the value at the pion mass shell is not directly accessible in experiments. For a recent analysis obtained from different experiments ( $\pi N$ scattering, $N N$ scattering, ...) see ref. [39]. In this respect there is certainly the need to calculate this observable from a more fundamental quark level. Within our approach the pion nucleon form factor is obtained by coupling an external pion current to the diquark-quark bound state using again Mandelstam's formalism. Because

of parity conservation one notes that there is no contribution from the loop integral (52) where the pion would couple to the $0^{+}$diquark. So the relevant nucleon current is completely carried by the quark part (51). In order to specify the vertex function for the pion-quark coupling we use spontaneously broken chiral symmetry and Goldstone's theorem [40]: In the chiral limit the vertex function, which is nothing else than the pion Bethe-Salpeter amplitude, is proportional to the scalar self energy of the quark. This is a consequence of the fact that in the chiral limit (vanishing quark current mass) where, provided the pion mass vanishes (Goldstone's theorem), the pion Bethe-Salpeter equation is equivalent to the quark Dyson-Schwinger equation. Therefore the proper choice is

$$
\Gamma_{\pi}^{a}\left(P_{\pi}^{2}=Q^{2}=0, p^{2}\right)=i \gamma_{5} \frac{B\left(p^{2}\right)}{f_{\pi}} \tau^{a},
$$

where $B\left(p^{2}\right)$ is defined in eq. (58) and the experimental pion decay constant $f_{\pi}=0.093$ $\mathrm{GeV}$ is used. Although this choice of the pion-quark vertex is valid only at the pion mass shell $Q^{2}=0$, we assume that the offshell amplitude does not vary too much with the pion momentum and therefore the most obvious generalization [41]

$$
\Gamma_{\pi}^{a}\left(Q^{2}, p^{2}\right)=i \gamma_{5} \frac{\tau^{a}}{2} \frac{1}{f_{\pi}}\left(B\left(\left(p-\frac{1}{2} Q\right)^{2}\right)+B\left(\left(p+\frac{1}{2} Q\right)^{2}\right)\right)
$$

of the onshell vertex function is allowed at least for a few hundert $\mathrm{MeV}$ around the onshell point.

When inserting the pion quark vertex function into eq. (51) and evaluating the loop integral we determine the pion-nucleon form factor. Furthermore since the pion-nucleon matrix element is parametrized as

$$
\begin{aligned}
J_{\pi}\left(Q^{2}\right) & =\left\langle N\left(P_{f}, S_{f}\right)\left|j_{\pi}\left(Q^{2}\right)\right| N\left(P_{i}, S_{i}\right)\right\rangle \\
& =\Lambda^{+}\left(P_{f}, S_{f}\right)\left[\gamma_{5} g_{\pi N N}\left(Q^{2}\right) \tau^{a}\right] \Lambda^{+}\left(P_{i}, S_{i}\right) .
\end{aligned}
$$

the extraction of $g_{\pi N N}\left(Q^{2}\right)$ is straightforward.

\subsection{Axial Form Factor}

Finally we calculate the axial form factor $g_{A}\left(Q^{2}\right)$ of the nucleon. Due to parity, we again have to consider only the quark part of the nucleon current, since an axial current does 
not couple to the scalar diquark. To specify the relevant vertex function, where the axial current interacts with the quark, we use the chiral Ward identity which reads

$$
Q_{\mu} \Gamma_{5 \mu}(p+Q, p)=S^{-1}(p+Q) \gamma_{5}-\gamma_{5} S^{-1}(p)
$$

This symmetry constraint can be satisfied with the vertex 40]

$$
\begin{aligned}
\Gamma_{5 \mu}^{a}(p, k) & =\left(i \frac{A\left(p^{2}\right)+A\left(k^{2}\right)}{2} \gamma_{\mu}\right. \\
& \left.+i \frac{(p+k)_{\mu}}{p^{2}-k^{2}}\left[\left(A\left(p^{2}\right)-A\left(k^{2}\right)\right) \frac{\gamma p+\gamma k}{2}-i\left(B\left(p^{2}\right)+B\left(k^{2}\right)\right)\right]\right) \gamma_{5} \frac{\tau^{a}}{2}
\end{aligned}
$$

that is totally determined by the quark self-energies. Although the axialvector vertex function looks, up to the isospin generators and the explicit $\gamma_{5}$, very similar to the BallChiu vertex (57) (used in the case of a vector current coupling), there is one important difference: In the last term the scalar quark self-energies $B\left(p^{2}\right)$ and $B\left(k^{2}\right)$ add up, which in the limit $Q=p-k \rightarrow 0$ leads to

$$
Q_{\mu} \Gamma_{5 \mu}^{a}(p, k) \longrightarrow \tau^{a} B\left(p^{2}\right) \gamma_{5}
$$

When comparing (70) with eq. (65), the pion-quark vertex, one observes the exact Goldberger Treiman relation for the quarks in the chiral limit:

$$
\lim _{Q \rightarrow 0} i Q_{\mu} \Gamma_{5 \mu}^{a}(p, k)=f_{\pi} \Gamma_{\pi}^{a}\left(0, p^{2}\right) .
$$

Stated in other words, in the chiral limit the axialvector quark coupling for vanishing momentum transfer is completely dominated by the pseudoscalar coupling to a massless pion (conserved axial current).

To examine whether the phenomenologically much more important Goldberger-Treiman relation on the nucleon level

$$
g_{\pi N N}=g_{A} \frac{M}{f_{\pi}}
$$

is fulfilled, we have to determine $g_{A}\left(Q^{2}\right)$ by taking the matrix element of the axial current between the diquark-quark bound states. After using the axialvector vertex function (69) to evaluate (51) and comparing the result with the familiar decomposition

$$
\begin{aligned}
J_{\mu}^{a}\left(Q^{2}\right) & =\left\langle N\left(P_{f}, S_{f}\right)\left|j_{\mu}^{a}\left(Q^{2}\right)\right| N\left(P_{i}, S\right)\right\rangle \\
& =\Lambda^{+}\left(P_{f}, S_{f}\right) \frac{\tau^{a}}{2}\left[\gamma_{\mu} g_{A}\left(Q^{2}\right)+Q_{\mu} g_{P}\left(Q^{2}\right)\right] \gamma_{5} \Lambda^{+}\left(P_{i}, S_{i}\right)
\end{aligned}
$$

of the matrix element, the invariant form factors are immediately accessible. Note that besides $g_{A}\left(Q^{2}\right)$, the axial form factor of the nucleon, there exist another form factor $g_{P}\left(Q^{2}\right)$, the induced pseudoscalar form factor with a pole at the pion mass shell. 


\subsection{Discussion of Numerical Results}

In this subsection we finally show and discuss our results for the nucleon form factors. We will concentrate again on three different cases: Using type I propagators, the solution of the corresponding Bethe-Salpeter equation and the appropriate vertex functions; using type II propagators with $d=1$ and with $d=10$. The so obtained electromagnetic form factors of the nucleon are shown in fig. (7).

$F_{e}{ }^{P}\left(Q^{2}\right)$

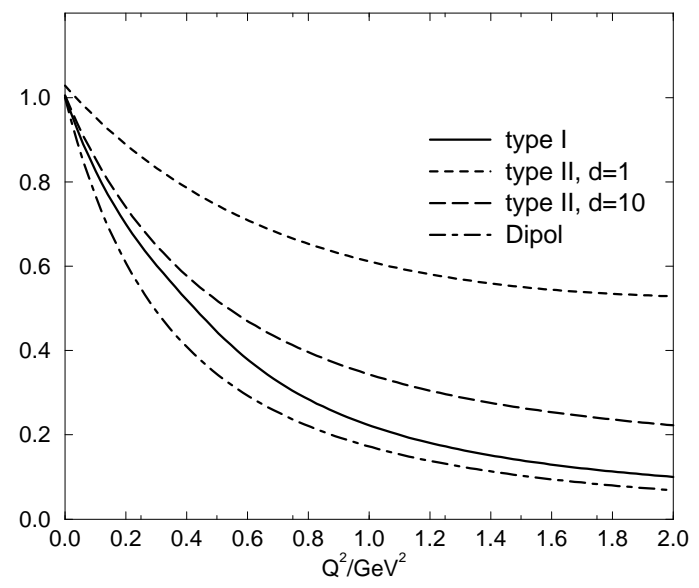

$F_{m}{ }^{P}\left(Q^{2}\right)$

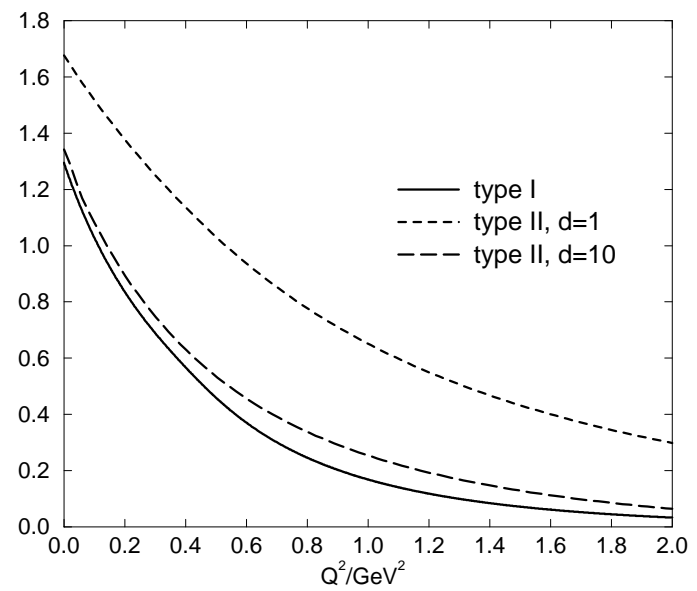

$\mathrm{F}_{\mathrm{e}}^{\mathrm{N}}\left(Q^{2}\right)$
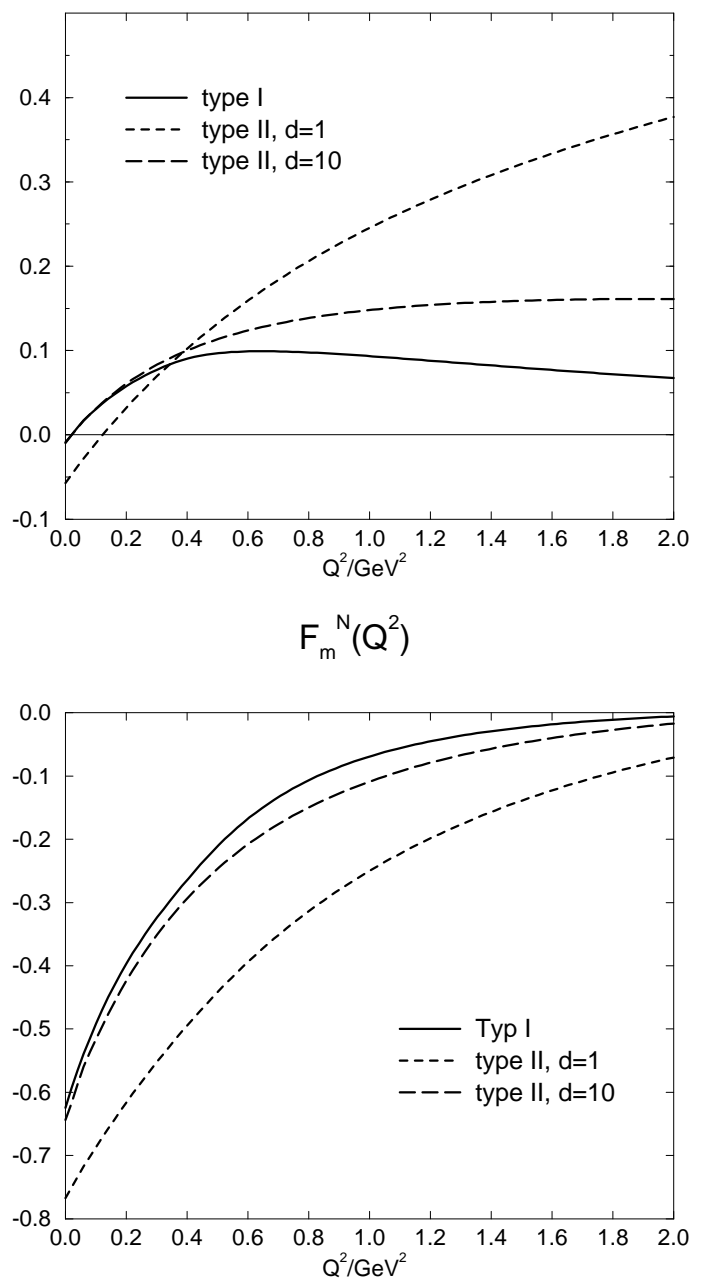

Fig. 7. Electromagnetic form factors of the nucleon.The nucleon mass in all cases is chosen to be $M=1.9 m_{q}$ and the amplitudes entering the calculation of the matrix elements are obtained with $\Lambda=2 m_{q}$.

First of all, we mention (as can also be seen in the figures), that it is not possible in general to obtain exact charge conservation. This is due to the impulse approximation, where the coupling of the photon to the exchanged quark (and also to the diquark-quark form factor) is neglected. Whereas in a Salpeter approach charge conservation can be 
shown analytically [32] to guarantee charge conservation of mesons within the Bethe-Salpeter approach [42], in our fully relativistic nucleon calculation the situation is more complicated (see also ref. [29]). But it can also be seen in the figures that the violation of charge conservation $\Delta Q_{P, N}$ is at the most only a few percent (due to isospin symmetry $\Delta Q_{N}=-2 \Delta Q_{P}$ ). In this respect the contribution of the neglected exchange graph is more important for the case of a minimal screening of the mass poles (type II with $d=1$ ).

Furthermore the electric form factor of the proton in calculations of type I and type II with $d=10$ are in reasonable correspondence with the empirical dipol fit

$$
F_{e}^{P}\left(Q^{2}\right)=\frac{1}{\left(1+Q^{2} / 0.71 \mathrm{GeV}^{2}\right)^{2}}
$$

The result obtained with $d=1$ falls short in this respect: The variation of $F_{e}^{P}\left(Q^{2}\right)$ with the photon momentum is too weak. This feature is also present for the other form factors. Obviously the modifications of the propagators with a pure exponentional factor severly changes the spacelike observable nucleon properties.

The electric neutron form factor shows a reasonable course. Note that a sensible description of neutron properties was one of the motivations for the diquark concept [43], having the following intuitive (nonrelativistic) picture in mind: A lighter quark circling around a heavier scalar diquark inevitably leads to a negative mean square radius of the neutron. It is now satisfying to observe that our relativistic approach, although working with equal masses of the constituents, confirmes this intuitive picture; it is not trivial at all, that the quark and diquark contributions give rise to a qualitatively correct behaviour of the electric neutron form factor.

\section{Table 3}

\begin{tabular}{||c||c|c|c|c||}
\hline \multicolumn{5}{||c||}{ Nucleon form factors } \\
\hline & type I & type II, $d=1$ & type II, $d=10$ & Exp. \\
\hline$Q_{P}$ & $1+4.1 \cdot 10^{-3}$ & $1+2.85 \cdot 10^{-2}$ & $1+4.7 \cdot 10^{-3}$ & 1 \\
$Q_{N}$ & $-8.2 \cdot 10^{-3}$ & $-5.70 \cdot 10^{-2}$ & $9.4 \cdot 10^{-3}$ & 0 \\
\hline$\mu_{P}$ & 1.32 & 1.68 & 1.34 & 2.79 \\
$\mu_{N}$ & -0.64 & -0.77 & -0.65 & -1.91 \\
\hline$g_{A}$ & 1.39 & 1.31 & 1.41 & 1.25 \\
$g_{\pi N N}$ & 10.59 & 14.17 & 10.89 & $13-14.5$ \\
\hline \hline
\end{tabular}

In this table we show the basic results for the form factors. Note that all magnetic moments are given in in units of $e / 2 M$. All displayed observables are obtained in calculations, where $S_{1}(p)$ and $S_{2}(p)$ were included. The number of considered Gegenbauer polynomials was terminated after convergence was achieved.

$\overline{7 \text { Going }}$ to the Salpeter limit we also get charge conservation numerically. 
Axial form factor $g_{A}\left(Q^{2}\right)$

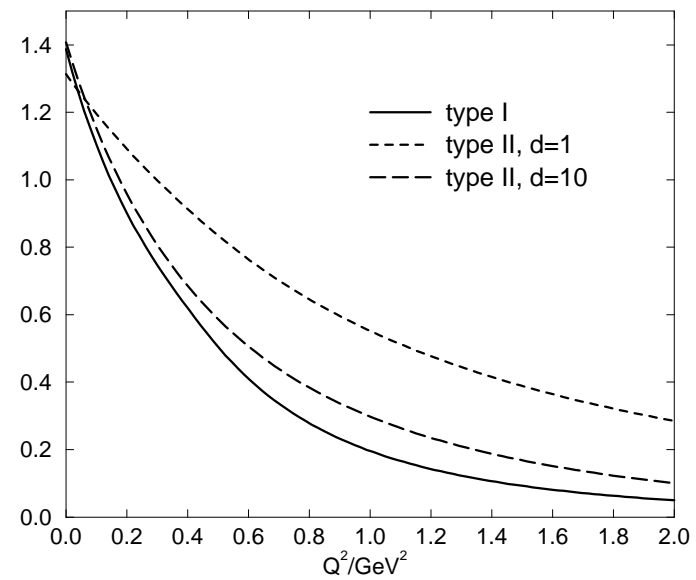

Pion nucleon form factor $g_{\pi N N}\left(Q^{2}\right)$

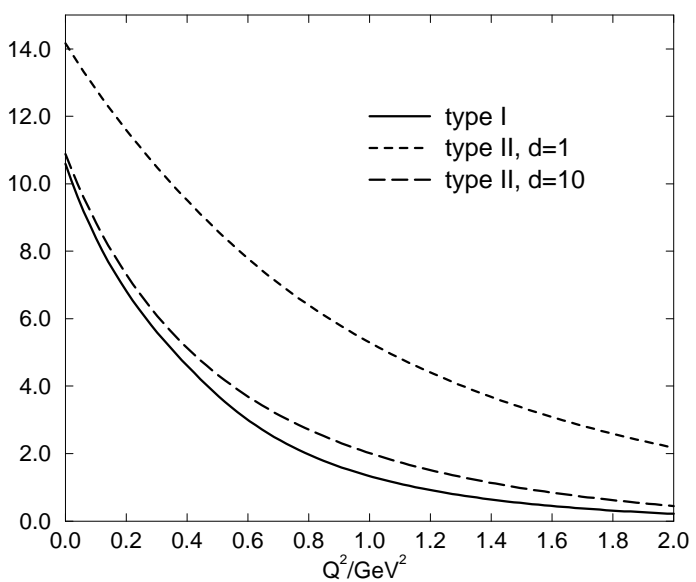

Fig. 8. The axial form factor $g_{A}\left(Q^{2}\right)$ of the nucleon and the pion nucleon form factor $g_{\pi N N}\left(Q^{2}\right)$ are shown for type I and type II with $d=1,10$ calculations respectively.

Axial form factor $g_{A}\left(Q^{2}\right)$

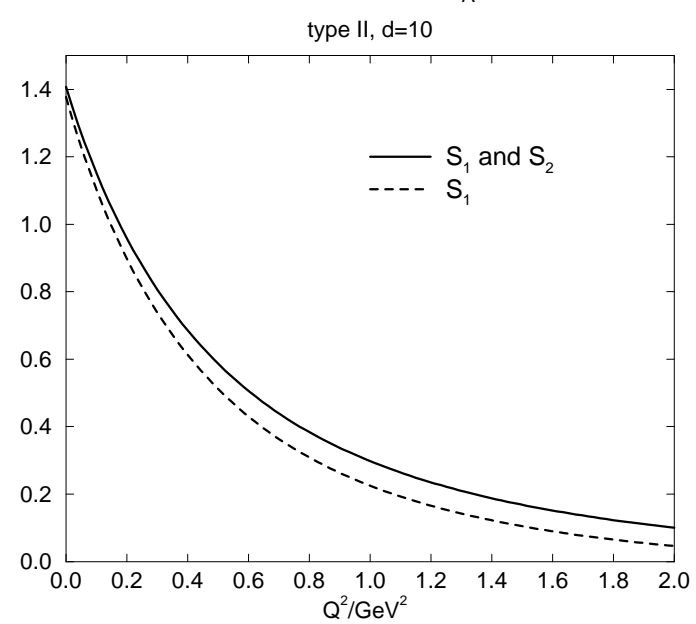

Pion nucleon form factor $g_{\pi N N}\left(Q^{2}\right)$

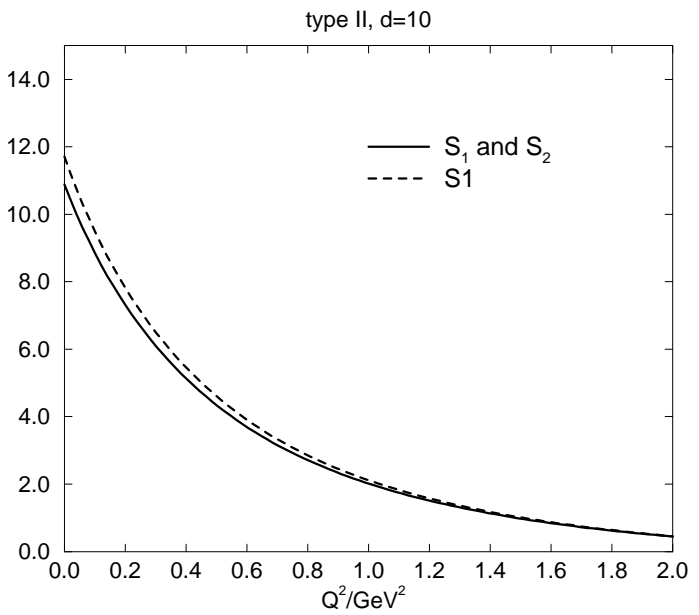

Fig. 9. The axial form factor $g_{A}\left(Q^{2}\right)$ and the pion nucleon form factor $g_{\pi N N}\left(Q^{2}\right)$ are shown for a type II with $d=10$ calculation, including only $S_{1}(p)$ or including $S_{1}(p)$ and $S_{2}(p)$.

For both proton and neutron, the magnetic moments are too small. This is, however, not surprising because large contributions coming from $1^{+}$diquarks are expected for these observables (32], 44]). Nevertheless, the calculated values have magnitudes expected from a calculation involving only $0^{+}$diquarks [30,31.

Before discussing the axial properties of the nucleon, we mention the following behaviour of the form factors: When $\Lambda$ is decreased, leading to narrower amplitudes in momentum space (compare fig. (函)), the electric form factors for the type I calculation become steeper and approach the emperical dipol fit. While such a behaviour is expected near threshold 45] we do not observe this feature for type II calculations. In these calculations the form factors (at leat at small $Q^{2}$ ) are very insensitive on $\Lambda$ and the actual value of the bound 
state mass.

Our results for the axial and the pion-nucleon form factor are shown in fig. (8). Again, we observe for a type II with $d=1$ calculation a relatively small variation of the form factors with $Q^{2}$. Furthermore for the calculation including type II propagators with $d=10$ the similarities to the type I case can be seen. A striking feature, however, is the good agreement of $g_{A}=g_{A}\left(Q^{2}=0\right)$ and $g_{\pi N N}=g_{\pi N N}\left(Q^{2}=0\right)$ with the experimental values. $g_{A}$ tends to be slightly too large whereas the pion nucleon coupling constant obtained with type I and with type II $(\mathrm{d}=10)$ is a little bit too small. Nevertheless, in all cases a qualitatively right behaviour is obtained. This is astonishing, because also for these observables (in analogy to the magnetic moments) sizeable contributions from $1^{+}$diquarks are expected. Note that in ref. [29] also a relatively large value of $g_{A}$ has been obtained, including only $0^{+}$diquarks.

By comparing the calculated values of $g_{\pi N N}$ with the ones obtained form the Goldberger Treiman relation (72) we observe a violation of the Goldberger Treiman relation up to $30 \%$. This failure also signals the necessity for the inclusion of $1^{+}$diquarks in the calculation. It is furthermore to be seen how much the exchanged quark contributes to these observables.

In figure (9) we show the influence of the lower component $S_{2}(p)$ of the nucleon Dirac field by comparing the results obtained with all amplitudes with the result obtained with $S_{1}(p)$ alone. It is interesting to note that the values of $g_{\pi N N}\left(Q^{2}\right)$ at small values of the momentum transfer are influenced by $S_{2}(p)$; while the values of $g_{A}\left(Q^{2}\right)$ at larger values are slightly more affected. Nevertheless we conclude that having a large lower component is probably only due to the representation chosen for the Dirac matrices. They do not have a significant effect on observables studied so far.

\section{Conclusions and Outlook}

In this paper we have developed a covariant diquark-quark model suitable for the calculation of nucleon observables. As discussed, confinement is put in by a modification of the free (tree-level) quark and diquark propagators. The structure of the Bethe-Salpeter equation, describing nucleons as diquark-quark bound states interacting through quark exchange, has been elaborated using an appropriate decomposition of the Bethe-Salpeter vertex function in the Dirac algebra. Furthermore, the coefficient functions of the corresponding Lorentz tensors have been expanded in hyperspherical harmonics, exploiting an approximate $O(4)$ symmetry (which would be exact if the exchanged particle were massless). After discussing the various numerical methods we presented our results for the calculation including $0^{+}$diquarks. The advantages of the confining propagators are clearly seen in the absence of unphysical thresholds.

The numerically obtained nucleon vertex function has been fitted to a simple analytic form and then used to calculate nucleon matrix elements and form factors. In particular, we considered electromagnetic, the axial and the pionic form factors of the nucleon. While, due to the various simplifications and approximations, a quantitative agreement of the 
results with experimental values could not be expected, we nevertheless got reasonable results. Furthermore, the large lower component of the nucleon Dirac field was shown to be merely an artefact of the chosen spinor representation. Although, the various observables displayed that the developed picture of a nucleon is still oversimplified, they nevertheless give us confidence that we are on the right track in obtaining a reasonable and trustable baryon model. The next step of improvement will be the inclusion of $1^{+}$diquarks in the Bethe-Salpeter equation and also in the nucleon matrix elements. Furthermore we plan to determine the internal structure of the diquarks (described crudely by the parameter $\Lambda$ ) by a microscopic diquark model.

In order to fully exploit the advantages and possibilities of our covariant and confining approach, the model will soon be applied to other processes. In particular, we will investigate the different observables associated with the reactions $p+\gamma \rightarrow K+\Lambda$ and $p+p \rightarrow K+\Lambda$ which are measured at ELSA [46] and COSY [47], respectively.

While in most calculations of nucleon structure functions the distribution functions of the constituents are merely parametrized (see e.g. 448), our approach offers the possibility to determine, in a first step, these distributions, after the constituents are specified. In ref. 24] this was investigated, assuming the scalar diquark to be a spectator and the photon interacts only with the quark.

We conclude, that the reported studies are a good starting point for further investigations, which certainly will improve our qualitative and quantitative understanding of the baryon structure.

Acknowledgments We thank Rolf Bäurle and Udo Zückert for their contributions in the early stages of this work, and G. Piller and K. Kusaka for the very helpful discussions on the numerical solution of the Bethe-Salpeter equation. Helpful remarks by F. Lenz are gratefully acknowleged.

\section{A Dirac Decomposition}

We choose the following ansatz for the amplitude $\chi^{\mu}$

$$
\begin{aligned}
\chi^{\mu}(P, p) & =A_{1}(P, p) \gamma_{5} \hat{P}^{\mu} \Xi \Lambda^{+}+A_{2}(P, p) \gamma_{5} \hat{P}^{\mu} \Lambda^{+} \\
& +\tilde{B}_{1}(P, p) \gamma_{5} \hat{p}_{T}^{\mu} \Xi \Lambda^{+}+\tilde{B}_{2}(P, p) \gamma_{5} \hat{p}_{T}^{\mu} \Lambda^{+} \\
& +\tilde{C}_{1}(P, p) \gamma_{5} i\left(\hat{P}^{\mu} \mathbf{1}-\gamma^{\mu}\right) \Lambda^{+}+\tilde{C}_{2}(P, p) \gamma_{5} i\left(\hat{P}^{\mu} \mathbf{1}+\gamma^{\mu}\right) \Xi \Lambda^{+}
\end{aligned}
$$

In the rest frame of the bound state, $P=(0,0,0, i M)$, we obtain by using

$$
\Lambda^{+}=\left(\begin{array}{cc}
1 & 0 \\
0 & 0
\end{array}\right), \quad \Xi \Lambda^{+}=\left(\begin{array}{cc}
0 & 0 \\
\hat{\boldsymbol{p}} \boldsymbol{\sigma} & 0
\end{array}\right)
$$

the following expressions:

$$
\chi^{4}=\left(\begin{array}{cc}
\hat{\boldsymbol{p}} \boldsymbol{\sigma} A_{1}(P, p) & 0 \\
\mathbf{1} A_{2}(P, p) & 0
\end{array}\right)
$$


and

$$
\chi=\left(\begin{array}{cc}
(\hat{\boldsymbol{p}} \boldsymbol{\sigma}) \hat{\boldsymbol{p}} \tilde{B}_{1}(P, p) & 0 \\
\hat{\boldsymbol{p}} \tilde{B}_{2}(P, p) & 0
\end{array}\right)+\left(\begin{array}{cc}
\boldsymbol{\sigma} \tilde{C}_{1}(P, p) & 0 \\
\boldsymbol{\sigma}(\hat{\boldsymbol{p}} \boldsymbol{\sigma}) \tilde{C}_{2}(P, p) & 0
\end{array}\right)
$$

Using the redefinitions

$$
\tilde{B}_{i}=B_{i}-C_{i}, \quad \tilde{C}_{i}=C_{i}
$$

turns eq. (A.1) into eq. (21). In the rest frame this leads to

$$
\chi=\left(\begin{array}{cc}
\hat{\boldsymbol{p}}(\hat{\boldsymbol{p}} \boldsymbol{\sigma}) B_{1}(P, p)+i(\boldsymbol{\sigma} \times \hat{\boldsymbol{p}})(\hat{\boldsymbol{p}} \boldsymbol{\sigma}) C_{1}(P, p) & 0 \\
\hat{\boldsymbol{p}} B_{2}(P, p)+i(\boldsymbol{\sigma} \times \hat{\boldsymbol{p}}) C_{2}(P, p) & 0
\end{array}\right)
$$

Thus in the rest frame the meaning of the amplitudes is therefore as follows: the amplitudes $A_{i}$ are the time components, the components $B_{i}$ are parallel to the relative three-momentum of the constituents, and the components in the third line in eq. (21) are orthogonal to the momentum and the spin.

\section{B Analytic fits of the amplitudes}

Fit Quality of $S_{10}(p)$

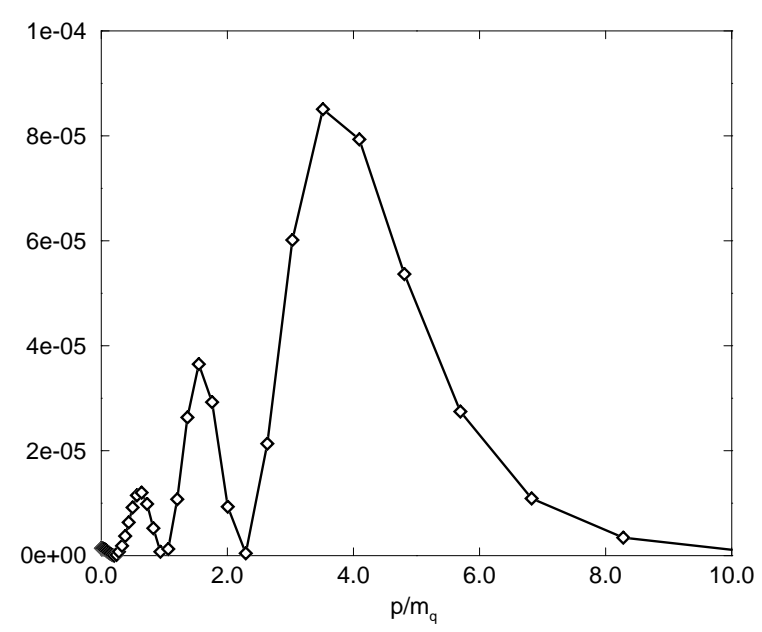

Fig. B.1. $\left(S_{10}(p)_{\text {numeric }}-S_{10}(p)_{\text {fit }}\right)^{2}$ is plotted for the type II calculation with $M=1.9 m_{q}$.

After solving the Bethe-Salpeter equation the amplitudes $S_{10}(p) \ldots S_{22}(p)$ are given only at the grid points of the momentum mesh. For further use in the calculation of matrix elements this a very unconvenient feature. Therefore we fit rational funtions to them. To do this the value of every amplitude at each grid point is therefore treated as a "data" point with a certain standart deviation $\sigma$. We find that the following set of rational functions are suitable to take the behaviour at small $p$ as well as the behaviour at large $p$ properly 


\begin{tabular}{||c||c|c|c|c|c|c||}
\hline \multicolumn{7}{||c||}{ Fit coefficients for type I } \\
\hline & $S_{10}$ & $S_{11}$ & $S_{12}$ & $S_{20}$ & $S_{21}$ & $S_{22}$ \\
\hline$a^{1}$ & 0.9992 & -0.6954 & 0.03028 & -0.4191 & 0.4113 & -0.0127 \\
$b^{1}$ & 0.3107 & 0.3028 & 0.4617 & 0.2682 & 0.1937 & 0.4090 \\
$a^{2}$ & -0.0002 & 0.2929 & 0.0134 & -0.1841 & -0.3939 & -0.0058 \\
$b^{2}$ & 7.8284 & 0.3028 & 0.1834 & 0.0792 & 0.1966 & 0.2085 \\
\hline$\chi^{2} \sigma$ & $6.786 \cdot 10^{-5}$ & $4.025 \cdot 10^{-5}$ & $9.474 \cdot 10^{-9}$ & $1.132 \cdot 10^{-5}$ & $2.347 \cdot 10^{-8}$ & $1.207 \cdot 10^{-10}$ \\
\hline
\end{tabular}

Table B.1

The fit coefficients for a type I calculation with $M=m_{q}$ and $\Lambda=2 m_{q}$ are displayed.

\begin{tabular}{||c||c|c|c|c|c|c||}
\hline \multicolumn{7}{||c||}{ Fit coefficients for type II, $d=1$} \\
\hline$a^{1}$ & 1.6562 & -0.9674 & 0.4988 & -0.4168 & 0.3898 & 0.5042 \\
$b^{1}$ & 0.2437 & 0.2556 & 0.1985 & 0.0924 & 0.1564 & 0.1562 \\
$a^{2}$ & -0.6574 & 0.6342 & -0.4686 & 0.0463 & -0.3528 & -0.5069 \\
$b^{2}$ & 0.3838 & 0.3293 & 0.2046 & 0.0924 & 0.1613 & 0.1558 \\
\hline$\chi^{2} \sigma$ & $5.305 \cdot 10^{-4}$ & $1.340 \cdot 10^{-4}$ & $5.331 \cdot 10^{-7}$ & $1.035 \cdot 10^{-4}$ & $5.263 \cdot 10^{-8}$ & $3.594 \cdot 10^{-9}$ \\
\hline
\end{tabular}

Table B.2

The fit coefficients for a type II calculation with $d=1, M=m_{q}$ and $\Lambda=2 m_{q}$ are displayed. into account :

$$
\begin{aligned}
& S_{10}(p)=\sum_{k=1}^{2} \frac{a_{10}^{k}}{\left(1+b_{10}^{k} p^{2}\right)^{2}} \\
& S_{11}(p)=\sum_{k=1}^{2} \frac{a_{11}^{k} p}{\left(1+b_{11}^{k} p^{2}\right)^{2}} \\
& S_{12}(p)=\sum_{k=1}^{2} \frac{a_{12}^{k} p^{2}}{\left(1+b_{12}^{k} p^{2}\right)^{3}} \\
& S_{20}(p)=\sum_{k=1}^{2} \frac{a_{20}^{k}}{\left(1+b_{20}^{k} p^{2}\right)^{3}} \\
& S_{21}(p)=\sum_{k=1}^{2} \frac{a_{21}^{k} p^{2}}{\left(1+b_{21}^{k} p^{2}\right)^{3}} \\
& S_{22}(p)=\sum_{k=1}^{2} \frac{a_{22}^{k} p^{3}}{\left(1+b_{22}^{k} p^{2}\right)^{4}}
\end{aligned}
$$


Note, that the momentum $p$ is taken in units of the quark mass. The fit coefficients $a_{i j}^{k}, b_{i j}^{k} ; i, j=1,2 ; k=1,2$ are then determined independently for each amplitude by performing a $\chi^{2}$ fit. To judge the quality of this procedure we show in fig. (B.1) the squared deviation

$$
\tilde{\Delta}^{2}=\left(S_{10}(p)_{\text {numeric }}-S_{10}(p)_{f i t}\right)^{2}
$$

Suming up the squared deviations then leads to

$$
\sigma^{2} \chi^{2}=\sum_{k=1}^{k \max } \Delta^{2}=\sum_{k=1}^{k \max }\left(S_{10}(k)_{n u m e r i c}-S_{10}(k)_{f i t}\right)^{2}
$$

i.e. the merit function $\chi^{2}$ times the squared accuracy of the "data points". In the tables (B.1) and (B.2) we show the fit coefficients which reproduce the plots for the type I and the type II with $d=1$ amplitudes given in figure (2) within very small deviations.

\section{Evaluation of the loop integrals}

In Section 4.1 we derived the two loop integrals building up the nucleon current. In eq. (51)

$$
J_{\mu}^{q}\left(Q^{2}\right)=\int \frac{d^{4} p}{(2 \pi)^{4}} \bar{\chi}\left(P_{f}, p_{f}\right) S\left(p_{+}\right) \Gamma_{\mu}^{q}\left(p_{+}, p_{-}\right) S\left(p_{-}\right) D\left(p_{d}\right) \chi\left(P_{i}, p_{i}\right),
$$

the momenta are definded as

$$
\begin{aligned}
p_{ \pm} & =p+\frac{1}{2} P_{i}+\frac{1}{2} Q \pm \frac{1}{2} Q \\
p_{d} & =-p+\frac{1}{2} P_{i}
\end{aligned}
$$

whereas in eq. (52)

$$
J_{\mu}^{d}\left(Q^{2}\right)=\int \frac{d^{4} p}{(2 \pi)^{4}} \bar{\chi}\left(P_{f}, p_{f}\right) D\left(p_{+}\right) \Gamma_{\mu}^{d}\left(p_{+}, p_{-}\right) D\left(p_{-}\right) S\left(p_{q}\right) \chi\left(P_{i}, p_{i}\right)
$$

the momenta are chosen as

$$
\begin{aligned}
p_{ \pm} & =-p+\frac{1}{2} P_{i}+\frac{1}{2} Q \pm \frac{1}{2} Q \\
p_{q} & =p+\frac{1}{2} P_{i}
\end{aligned}
$$

As stated in the text, to evaluate these integrals it is useful to work in the Breit-frame

$$
\begin{aligned}
Q & =\left(0,0, Q_{3}, 0\right) \\
P_{i} & =\left(0,0,-\frac{1}{2} Q_{3}, i \sqrt{M^{2}+\frac{1}{4} Q^{2}}\right) \\
P_{f} & =\left(0,0, \frac{1}{2} Q_{3}, i \sqrt{M^{2}+\frac{1}{4} Q^{2}}\right)
\end{aligned}
$$


and further to express the loop momentum $p$ in 4-dimensional spherical coordinates

$$
p=p(\sin \phi \sin \theta \cos \psi, \sin \phi \sin \theta \sin \psi, \sin \psi \cos \theta, \cos \psi) .
$$

While the $\phi$ integration is trivial, the integration over $p, \Theta$ and $\psi$ has to be performed numerically.

\section{References}

[1] Procceedings of the Conference Diquarks 3, Torino, Oct. 28-30,1996; eds.: M. Anselmino and E. Predazzi, to be published by World Scientific.

[2] R. T. Cahill, Austr. J. Phys. 42, 171 (1989).

[3] H. Reinhardt, Phys. Lett. B244, 316 (1990).

[4] M. G. Olsson, e-print hep-ph/9702213.

[5] P. Hasenfratz and J. Kuti, Phys. Rep. 40, 75 (1978).

[6] T. H. R. Skyrme, Proc. Roy. Soc. A260, 127 (1961); see also: G. S. Adkins, C. R. Nappi, and E. Witten, Nucl. Phys. B228, 552 (1983); G. Holzwarth (Ed.), Baryons as Skyrme Solitons (World Scientific Publ. Comp., Singapore, 1993).

[7] R. Alkofer, H. Reinhardt, and H. Weigel, Phys. Rep. 265, 139 (1996);

C. V. Christov et al., Prog. Part. Nucl. Phys. 37, 1 (1996).

[8] M. Gell-Mann, Phys. Rev. 8, 214 (1964).

[9] G. Karl and E. Obryk, Nucl. Phys. B8, 609 (1968).

[10] D. Faiman and A. W. Hendry, Phys. Rev. 173, 1720 (1968).

[11] L. Ya. Glozman, Z. Papp, W. Plessas, K. Varga and R. F. Wagenbrunn, e-print nucl-th/9705011.

[12] R. P. Feynman, M. Kislinger, and F. Ravndal, Phys. Rev. D3, 2706 (1971).

[13] A. Chodos and C. Thorn, Phys. Rev. D12, 2733 (1975).

[14] M. Rho, Phys. Rep. 240, 1 (1994).

[15] Y. Nambu and G. Jona-Lasinio, Phys. Rev. 122, 345 (1961).

[16] U. Zückert, R. Alkofer, H. Weigel, and H. Reinhardt, Phys. Lett. B362, 1 (1995); Phys. Rev. C55, 2030 (1997).

[17] H. J. Munczek and A. M. Nemirovsky, Phys. Rev. D 28, 181 (1983).

[18] D. Ebert, T. Feldmann and H. Reinhardt, Phys. Lett. B388, 154 (1996).

[19] A. Bender, C. D. Roberts, and L. v. Smekal, Phys. Lett. B380, 7 (1996). 
[20] P. Jain and H. J. Munczek, Phys. Rev. D 48 (1993) 5403.

[21] A. Buck, R. Alkofer, and H. Reinhardt, Phys. Lett. B286, 29 (1992);

A. Buck and H. Reinhardt, Phys. Lett. B356, 168 (1995).

[22] C. Hanhart and S. Krewald, Phys. Lett. B344, 55 (1995).

[23] G. Hellstern, R. Alkofer and H. Reinhardt, in preparation.

[24] K. Kusaka, G. Piller, A. W. Thomas, A. G. Williams, e-print hep-ph/9609277, to be published in Phys. Rev. (1997).

[25] H. Meyer, Phys. Lett. B337, 37 (1994).

[26] M. Abramowitz and I. A. Stegun, Handbook of Mathematical Functions, Dover, New York, 1965 .

[27] V. Keiner, Z. Phys. A354, 87 (1996).

[28] N. Ishii, W. Bentz, and K. Yazaki, Nucl. Phys. A587, 617 (1995).

[29] H. Asami, N. Ishii, W. Bentz and K. Yazaki, Phys. Rev. C 52, 3388 (1996).

[30] G. Hellstern, C. Weiss, Phys. Lett. B 35164 (1995).

[31] V. Keiner, Z. Phys. A354, 87 (1996).

[32] V. Keiner, Phys. Rev. C 54, (1996) 3232;

PhD Thesis, University of Bonn 1997, http://pythia.itkp.uni-bonn.de/bspub.htm].

[33] S. Mandelstam, Proc. Roy. Soc. 233248 (1955).

[34] C. Itzykson, J.-B. Zuber: Quantum Field Theory, McGraw-Hill (1985).

[35] J. S. Ball and T.W. Chiu, Phys. Rev. D 222542 (1980).

[36] D. Kusno, Phys. Rev. D 271657 (1983).

[37] D. C. Curtis and M. R. Pennington, Phys. Rev. D46 2663 (1992).

[38] K. Ohta, Phys. Rev. C 411213 (1990).

[39] T. E. O. Ericson, Nucl. Phys. A 543 409c (1993);

T. E. O. Ericson and B. Loiseau, hep-ph/9612420.

[40] R. Delbourgo, M. D. Scadron, J. Phys. G 5 (1979) 1621.

[41] C. D. Roberts, A. G. Williams, Prog. Part. Nuc. Phys. 33 (1994).

[42] C. D. Roberts, Nucl. Phys. A 605 (1996) 475.

[43] Z. Dziembowski, W. J. Metzger and R. T. Van de Walle, Z. Phys. C 10231 (1981).

[44] C. Weiss, A. Buck, R. Alkofer and H. Reinhardt, Phys. Lett. B 312 (1993) 6.

[45] R. L. Jaffe, P. F. Mende, Nucl. Phys. B369 (1992) 189.

[46] M. Bockhorst et. al. (SAPHIR), Z. Phys. C63, (1994) 37.

[47] W. Eyrich, private communication.

[48] R. Jakob, P. J. Mulders and J. Rodrigues, e-print hep-ph/9704335. 